\title{
A reduced basis method for multiple electromagnetic scattering in three dimensions
}

\author{
M. Ganesh ${ }^{\mathrm{a}}$, J.S. Hesthaven ${ }^{\mathrm{b}}$, B. Stamm ${ }^{\mathrm{c}}$ \\ ${ }^{a}$ Department of Mathematical and Computer Sciences, Colorado School of Mines, Golden, CO 80401 \\ mganesh@mines.edu \\ ${ }^{b}$ Division of Applied Mathematics, Brown University, Box F, Providence, RI 02912 \\ Jan. Hesthaven@brown . edu \\ ${ }^{c}$ Department of Mathematics, University of California, Berkeley, CA 94720 \\ stamm@math. berkeley.edu
}

\begin{abstract}
We consider the development of efficient and fast computational methods for parametrized electromagnetic scattering problems involving many scattering bodies. The parametrization may describe the location, orientation, size and shape of the scattering bodies as well as properties of the source field such as frequency, polarization and incident angle. The emphasis is on problems that need to be solved rapidly to accurately evaluate the scattering under parametric variation, e.g., for design, detection, or uncertainty quantification. For such problems, the use of a brute force approach is often ruled out due to the computational cost associated with solving the problem for each parameter value.

In this work, we show that the use of a reduced basis method based for a boundary element method for few reference scatterer configurations allows us to formulate a rapidly converging iterative method to resolve the computationally challenging large scale problem. The approach includes (i) a computationally intensive offline procedure to create a selection of a set of snapshot parameters and the construction of an associated reduced basis for each reference scatterer configuration and (ii) an inexpensive online algorithm to generate the surface current and scattering of the parametrized configuration, for any choice of parameters within the parameter domains used in the offline procedure. Comparison of our numerical results with directly measured results for some benchmark configurations demonstrate the power of our method to rapid evaluate the scattering under parametric variation of the overall configuration.
\end{abstract}

Keywords: Reduced basis, Multiple scattering, electromagnetic scattering, Maxwell equations, surface integrals, boundary element

2000 MSC: 65R20, 65N35. 


\section{Introduction}

The need for fast and efficient modeling of the scattering of electromagnetic waves by multiple particles is fundamental to several applications, see [1] and references therein. However, the direct modeling of such problems is both costly and complex due to the many scatterers and their individual interactions. Furthermore, such problems are particularly challenging when one seeks to evaluate the scattering under variations of the overall problem, i.e., with variations in frequency, direction and polarization of the incident waves, and the location, orientation, and even number of the particles. Attempting to evaluate the impact of such variations are often not practical using brute-force techniques, yet has many important applications in design, optimization, and inverse problems.

In such cases, it may be attractive and often required to seek a different approach and attempt to precompute and store scattering information of the configuration for a relative small representative set of parameters (using some offline process) and then develop a tool for the rapid online simulations that require the solution of substantially reduced dimensional systems for any chosen parameter value. This line of argument is similar to that leading to the reduced basis method, gaining increasing interest in the broader computational science community.

The basic idea of a reduced basis method was first introduced in the 1970's for nonlinear structural analysis $[2,3]$ and it was subsequently abstracted, analyzed $[4,5]$ and generalized to other type of parametrized partial differential equations [6,7]. Most of these earlier works focus on arguments that are local in the parameter space. Expansions are typically defined around a particular point of interest and the associated a priori analysis relies on asymptotic arguments on sufficiently small neighborhoods $[8,9]$. In such cases, the computational improvements are quite modest. In $[10,11]$ a global approximation space was built by using solutions of the governing PDE at globally sampled points in the parameter space, resulting in a much more efficient method. However, no a priori theory or a posteriori error estimators were developed in this early work.

In recent years, a number of novel ideas and essentially new features have been presented $[12,13,14,15,16$, $17,18,19,20]$. In particular, global approximation spaces are used and uniform exponential convergence of the reduced basis approximation has been numerically observed and confirmed in [21] where the first theoretical a priori convergence result for a one dimensional parametric space problem is presented. The development of rigorous a posteriori error estimators has also been presented, thereby transforming the reduced basis methods from an experimental technique to a computational method with a true predictive value.

While the vast majority of past work has focused on the development of methods for parametrized partial differential equations, recent activities has extended this approach [22] to the case of integral formulations and the electric field integral equations. For fixed parameters, multiple electromagnetic scattering algorithms have been discussed [23].

In this work we shall seek to further extend the use of reduced basis methods to not only accelerate the prediction of the scattering by a single obstacle but also to develop a reduced basis approach to account for the interaction between scatterers. The approach taken is to (i) first solve (offline) the full electromagnetic scattering problem on one or a small number of reference obstacle(s) for some appropriate number of parameters. Further, using affine transformations from one of the reference shapes to each of the other scatterers, we construct small operators that describe the interaction between the scatterers in the configurations. Then (ii) for any (online) choice of the parameters, we demonstrate how the combination of this model and an iterative algorithm, requiring the solution of small linear systems allows one to compute the surface currents on the entire set of scatterers and hence to compute the functional output of the multiple scattering model.

While the offline process requires the solution $N$ large dense complex linear systems with dimension $\mathcal{N}>>N, \mathcal{N}$ being the dimension of the boundary element solver required to solve the problem at sufficient accuracy, the relative inexpensive online process provides an efficient way to simulate the electromagnetic scattering interaction in the multiple scatterer configuration for many choice of parameters by solving only reduced dimensional systems at a low computational cost.

What remains of the paper is organized as follows. In Section 2 we provide a general description of the scattering problem and the integral equations being discussed in this work. This sets the stage for Section 3 where we introduce the boundary element formulation of the scattering problem, including a detailed discussion of transformations between reference and physical scatterers. In Section 4 we introduce the reduced basis method for both the single and multiple scatterer problem and introduces the iterative approach that enables the computation of the global scattering from knowledge of the local scattering problem. A central element of the efficiency of the reduced basis methods 
is the assumption that all operators and sources are affine in the parameter. This is what allows the offline-online separation. However, as we discuss in detail in Section 5, this assumption is not satisfied for the integral equation formulation presented here and we discuss in detail how to overcome this by the use of empirical interpolation methods. This sets the stage for Section 6 where we present a number of three-dimensional computational experiments of increasing complexity, highlighting the robustness and versatility of the proposed method. Section 7 concludes with a few remarks and a discussion of future directions of research.

\section{General problem description}

In this work, we consider a general three-dimensional electromagnetic scatterer, assumed to consist of disjoint perfect conducting obstacles $\Omega_{1}, \Omega_{2}, \ldots, \Omega_{\mathrm{J}}$, situated in a homogeneous medium with vanishing conductivity. We assume a time-harmonic plane wave exitasion by an incident electromagnetic wave $\left[\boldsymbol{E}^{\text {inc }}, \boldsymbol{H}^{\text {inc }}\right]$, (parameterized by the wavenumber $k \in \mathbb{R}^{+}$, the incident direction $\widetilde{\boldsymbol{d}}_{\text {inc }} \in S^{2}$ and polarization $\boldsymbol{p} \in \mathbb{R}^{3}$ ) impinging on the configuration $\Omega=\bigcup_{j=1}^{J} \Omega_{j}$.

The input vector fields $\boldsymbol{E}^{\text {inc }}, \boldsymbol{H}^{\text {inc }}:\left(\mathbb{R}^{3} \backslash \bar{\Omega}\right) \times \mathbb{R}^{+} \times S^{2} \times \mathbb{R}^{3} \rightarrow \mathbb{C}^{3}$ are, respectively, the incident electric and magnetic fields with wavelength $\lambda=2 \pi / k$. Here, $S^{2}$ is the unit sphere in $\mathbb{R}^{3}$ and $k=\omega \sqrt{\mu_{0} \epsilon_{0}}$, with $\omega>0$ being the angular frequency. Here we introduce the free space permittivity $\epsilon_{0}=10^{7} /\left(4 \pi c^{2}\right) \mathrm{F} / \mathrm{m}$ and permeability $\mu_{0}=4 \pi \times 10^{-7} \mathrm{H} / \mathrm{m}$, where $c=299,792,458 \mathrm{~m} / \mathrm{s}$ is the speed of light.

For a fixed wavenumber $k \in \mathbb{R}^{+}$, incident direction $\widetilde{\boldsymbol{d}}_{\text {inc }} \in S^{2}$, and polarization $\boldsymbol{p}_{\text {inc }} \in \mathbb{R}^{3}$, we take $\left[\boldsymbol{E}^{\text {inc }}, \boldsymbol{H}^{\text {inc }}\right]$ to be a plane wave with representation

$$
\boldsymbol{E}^{\mathrm{inc}}(\boldsymbol{x} ; \boldsymbol{\mu})=\boldsymbol{p}_{\mathrm{inc}} e^{i k \boldsymbol{x} \cdot \widetilde{\boldsymbol{d}}_{\mathrm{inc}}}, \quad \boldsymbol{H}^{\mathrm{inc}}(\boldsymbol{x} ; \boldsymbol{\mu})=\left[\widetilde{\boldsymbol{d}}_{\mathrm{inc}} \times \boldsymbol{p}_{\mathrm{inc}}\right] e^{i k \boldsymbol{x} \cdot \widetilde{\boldsymbol{d}}_{\mathrm{inc}}}, \quad \widetilde{\boldsymbol{d}}_{\mathrm{inc}} \perp \boldsymbol{p}_{\mathrm{inc}},
$$

where, in this work, $\boldsymbol{\mu}=\left(k, \widetilde{\boldsymbol{d}}_{\text {inc }}, \boldsymbol{p}_{\text {inc }}\right) \in \mathcal{D}:=\left[k^{-}, k^{+}\right] \times S^{2} \times P \subset \mathbb{R}^{7}$, with $P$ being a bounded polarization vector domain in $\mathbb{R}^{3}$. Throughout the paper, we use $\sim$ to represent unit vectors and - for elements in a reference obstacle.

For each fixed parameter $\boldsymbol{\mu}=\left(k, \widetilde{\boldsymbol{d}}_{\text {inc }}, \boldsymbol{p}_{\text {inc }}\right) \in \mathcal{D} \subset \mathbb{R}^{7}$, the interacting time-harmonic electromagnetic waves, with frequency $\omega$ scattered by the ensemble $\Omega=\bigcup_{j=1}^{j} \Omega_{j}$ are described by the electric field $\mathcal{E}$ and magnetic field $\mathcal{H}$,

$$
\mathcal{E}(\boldsymbol{x}, t)=\operatorname{Re}\left\{\boldsymbol{E}(\boldsymbol{x}) e^{-i \omega t}\right\}, \quad \boldsymbol{H}(\boldsymbol{x}, t)=\operatorname{Re}\left\{\boldsymbol{H}(\boldsymbol{x}) e^{-i \omega t}\right\}, \quad \boldsymbol{x} \in \mathbb{R}^{3} \backslash \bar{\Omega} .
$$

The spatial components $[\boldsymbol{E}, \boldsymbol{H}]$ of the electromagnetic wave satisfy the time-harmonic Maxwell equations [24]:

$$
\operatorname{curl} \boldsymbol{E}(\boldsymbol{x})-i \omega \mu_{0} \boldsymbol{H}(\boldsymbol{x})=\mathbf{0}, \quad \boldsymbol{c u r l} \boldsymbol{H}(\boldsymbol{x})+i \omega \epsilon_{0} \boldsymbol{E}(\boldsymbol{x})=\mathbf{0}, \quad \boldsymbol{x} \in \mathbb{R}^{3} \backslash \bar{\Omega},
$$

and the Silver-Müller radiation condition

$$
\lim _{|\boldsymbol{x}| \rightarrow \infty}[\boldsymbol{H}(\boldsymbol{x}) \times \boldsymbol{x}-|\boldsymbol{x}| \boldsymbol{E}(\boldsymbol{x})]=\mathbf{0} .
$$

Using the first equation in (2.3), $\boldsymbol{H}$ can be computed using $\boldsymbol{E}$ and with $k=\omega \sqrt{\mu_{0} \epsilon_{0}}, Z=\sqrt{\mu_{0} / \epsilon_{0}}$ being the intrinsic impedance of vacuum (so that $k Z=\omega \mu_{0}, k / Z=\omega \epsilon_{0}$ ), it is easy to see from (2.3)-(2.4) that the electric field $\boldsymbol{E}$ satisfies

$$
\begin{aligned}
\operatorname{curl} \operatorname{curl} \boldsymbol{E}(\boldsymbol{x})-k^{2} \boldsymbol{E}(\boldsymbol{x}) & =\mathbf{0}, \quad \boldsymbol{x} \in \mathbb{R}^{3} \backslash \bar{\Omega}, \\
\lim _{|\boldsymbol{x}| \rightarrow \infty}[\operatorname{curl} \boldsymbol{E}(\boldsymbol{x}) \times \boldsymbol{x}-i k Z|\boldsymbol{x}| \boldsymbol{E}(\boldsymbol{x})] & =\mathbf{0} .
\end{aligned}
$$

Further the electric field satisfies inhomogeneous boundary condition [24]:

$$
\left(\pi_{T} \boldsymbol{E}\right)(\boldsymbol{x})=-\left(\pi_{T} \boldsymbol{E}^{\mathrm{inc}}\right)(\boldsymbol{x} ; \boldsymbol{\mu}), \quad \boldsymbol{x} \in \bigcup_{\mathrm{j}=1}^{\mathrm{J}} \partial \Omega_{\mathrm{j}},
$$

where $\pi_{T}$ is the tangential component trace operator defined for $x \in \partial \Omega_{j}, j=1, \ldots, J$, by $\left(\pi_{T} v\right)(x)=\mathrm{n}(\boldsymbol{x}) \times[v(x) \times$ $\mathrm{n}(\boldsymbol{x})$ ]; where for $\boldsymbol{x} \in \partial \Omega_{j}, \mathrm{n}(\boldsymbol{x})$ denotes the unit outward normal at $\boldsymbol{x}$ on the surface $\partial \Omega_{j}$ of the obstacle $\Omega_{j}$. This boundary condition (2.7) follows from the assumption that each obstacle $\Omega_{j}$ in the ensemble is a perfect conductor 
and hence the tangential component of the total electric field $\boldsymbol{E}+\boldsymbol{E}^{\text {inc }}$ vanishes on the ensemble surface. In (2.7), for $x \in \partial \Omega_{j}, \mathrm{n}(\boldsymbol{x})$ denotes the unit outward normal at $\boldsymbol{x}$ on the surface $\partial \Omega_{j}$ of the obstacle $\Omega_{j}$.

For properties of $\pi_{T}$ and for definition of the spaces $\boldsymbol{H}^{-1 / 2}\left(\operatorname{div}_{\partial \Omega}, \partial \Omega\right)$ and $\boldsymbol{H}^{-1 / 2}\left(\operatorname{curl}{ }_{\partial \Omega}, \partial \Omega\right)$ used in this work, we refer to [25, Theorem 4.1] and related discussions in [25, 26, 24] of such spaces for smooth, polyhedral, and Lipschitz domains, $\Omega$. These two spaces are mutually adjoint with respect to the scalar product (denoted throughout the paper by $\langle\cdot, \cdot\rangle)$ in $\boldsymbol{L}_{t}^{2}(\partial \Omega)^{2}:=\left\{\boldsymbol{v} \in\left[L^{2}(\partial \Omega)\right]^{3}: \boldsymbol{v} \cdot \mathrm{n}=0\right\}$,

The main aim of this work is to consider the varying parameter case and hence we use the notation $[\boldsymbol{E}(\boldsymbol{x} ; \boldsymbol{\mu}), \boldsymbol{H}(\boldsymbol{x} ; \boldsymbol{\mu})]$ to denote the scattered electromagnetic field generated exterior to the multiple particle configuration $\Omega$, for $x \in \mathbb{R}^{3} \backslash \bar{\Omega}$ and $\boldsymbol{\mu} \in \mathcal{D}$. An important component in analyzing the interaction of the electromagnetic waves with complex scatterers is the computation of the radar cross section (RCS) of the ensemble $\Omega$ measured by a receiver situated in the direction $\widetilde{\boldsymbol{d}}_{\text {rcs }}$. The RCS, denoted by $\sigma\left(\widetilde{\boldsymbol{d}}_{\text {rcs }} ; \boldsymbol{\mu}\right)$, measured in decibels (db) can be represented as [24]:

$$
\sigma\left(\widetilde{\boldsymbol{d}}_{\mathrm{rcs}}, \boldsymbol{\mu}\right)=10 \log _{10}\left(4 \pi\left|\boldsymbol{E}_{\infty}\left(\widetilde{\boldsymbol{d}}_{\mathrm{rcs}} ; \boldsymbol{\mu}\right)\right|^{2} /\left|\boldsymbol{p}_{\mathrm{inc}}\right|^{2}\right), \quad \boldsymbol{E}_{\infty}\left(\widetilde{\boldsymbol{d}}_{\mathrm{rcs}}, \boldsymbol{\mu}\right)=\lim _{r \rightarrow \infty} \boldsymbol{E}\left(r \widetilde{\boldsymbol{d}}_{\mathrm{rcs}}, \boldsymbol{\mu}\right) e^{-i k r} r,
$$

where $\boldsymbol{E}_{\infty}$ is the electric far field. Two types of RCS are of particular interest: (i) The RCS for all directions $\widetilde{\boldsymbol{d}}_{\mathrm{rcs}}$, with a fixed incident direction $\widetilde{\boldsymbol{d}}_{\text {inc }}$; (ii) The RCS for all directions $\widetilde{\boldsymbol{d}}_{\text {rcs }}$, with varying incident directions $\widetilde{\boldsymbol{d}}_{\text {inc }}=-\widetilde{\boldsymbol{d}}_{\text {rcs }}$. These are the bistatic and monostatic RCS respectively. Thus, in general the parameter set $\mathcal{D}$ is further enriched by $\widetilde{\boldsymbol{d}}_{\mathrm{rcs}} \in S^{2}$.

For a fixed frequency, co-location of the transmitter and receiver in the monostatic RCS leads to, in contrast to the bistatic case, the requirement of solving the Maxwell equations over a significant range of incident directions and polarizations varying in $S^{2} \times \mathbb{R}^{3}$. In the more complex case of transmitters at an unknown or propagating location, incident waves with various frequencies, directions and polarizations to test the reaction of the configuration requires one to solve the full three dimensional scattering Maxwell system for a very high number of parameters varying in $\mathcal{D} \subset \mathbb{R}^{7}$.

In this work we seek to avoid this substantial computational burden by solving the Maxwell system for only a reduced but carefully chosen set of $N$ parameters in $\mathcal{D}$ for each reference shape and provide the end user the flexibility to solve the Maxwell system for any parameter in $\mathcal{D}$, any choice of parameters describing the scatterers, and any choice of receiver direction, with a computational cost that depends solely on $N$.

\subsection{The electric field integral equation}

The electric far field required in the scattering calculation can be represented as a surface integral on the scattering ensemble [24] given by:

$$
\boldsymbol{E}_{\infty}\left(\widetilde{\boldsymbol{d}}_{\mathrm{rcs}}, \boldsymbol{\mu}\right)=\boldsymbol{E}_{\infty}\left(\boldsymbol{w} ; \widetilde{\boldsymbol{d}}_{\mathrm{rcs}}, \boldsymbol{\mu}\right)=\frac{i k Z}{4 \pi} \int_{\partial \Omega} \widetilde{\boldsymbol{d}}_{\mathrm{rcs}} \times\left[\boldsymbol{w}(\boldsymbol{x} ; \boldsymbol{\mu}) \times \widetilde{\boldsymbol{d}}_{\mathrm{rcs}}\right] e^{i k x \cdot \widetilde{\boldsymbol{d}}_{\mathrm{rcs}}} d s(\boldsymbol{x}),
$$

provided that the surface current $\boldsymbol{w}$ is known on the ensemble. The scattered electric field $\boldsymbol{E}$ satisfying (2.5)-(2.7) can be represented in terms of the surface current $\boldsymbol{w}$, as [24]

$$
\boldsymbol{E}(\boldsymbol{x}, \boldsymbol{\mu})=\boldsymbol{E}(\boldsymbol{w} ; \boldsymbol{x}, \boldsymbol{\mu})=i k Z\left(\mathbf{S}_{\mathrm{e}} \boldsymbol{w}\right)(\boldsymbol{x} ; \boldsymbol{\mu}), \quad \boldsymbol{x} \in \mathbb{R}^{3} \backslash \bar{\Omega},
$$

where, for a surface density $\boldsymbol{a} \in \boldsymbol{H}^{-1 / 2}\left(\operatorname{div}_{\partial \Omega}, \partial \Omega\right)$, the electric field operator $\mathbf{S}_{\mathrm{e}}$ is defined as [24]

$$
\left(\mathbf{S}_{\mathrm{e}} \boldsymbol{a}\right)(\boldsymbol{x} ; \boldsymbol{\mu})=\int_{\partial \Omega}\left[\Phi(\boldsymbol{x}, \boldsymbol{y}) \boldsymbol{a}(\boldsymbol{y})+\frac{1}{k^{2}} \operatorname{grad}_{x} \Phi(\boldsymbol{x}, \boldsymbol{y}) \operatorname{div}_{\boldsymbol{y}} \boldsymbol{a}(\boldsymbol{y})\right] d s(\boldsymbol{y}) .
$$

Here

$$
\Phi(\boldsymbol{x}, \boldsymbol{y})=\frac{1}{4 \pi} \frac{e^{i k|\boldsymbol{x}-\boldsymbol{y}|}}{|\boldsymbol{x}-\boldsymbol{y}|}
$$

is the fundamental solution of the Helmholtz equation with wavenumber $k$. By applying the tangential component trace operator $\pi_{T}$ in (2.10) and using the boundary condition (2.7), we obtain the electric field surface integral equation for the unknown surface current $\boldsymbol{w}(\boldsymbol{\mu})=\boldsymbol{w}(\cdot ; \boldsymbol{\mu}) \in \boldsymbol{H}^{-1 / 2}\left(\operatorname{div}_{\partial \Omega}, \partial \Omega\right)$ :

$$
i k Z\left(\mathbf{T}_{\mathrm{e}} \boldsymbol{w}\right)(\boldsymbol{x} ; \boldsymbol{\mu})=-\left(\pi_{T} \boldsymbol{E}^{\mathrm{inc}}\right)(\boldsymbol{x} ; \boldsymbol{\mu}), \quad \boldsymbol{x} \in \partial \Omega, \quad \boldsymbol{\mu} \in \mathcal{D} \backslash \mathcal{K}(\Omega),
$$


where $\mathbf{T}_{\mathrm{e}}=\pi_{T} \circ \mathbf{S}_{\mathrm{e}}: \boldsymbol{H}^{-1 / 2}\left(\operatorname{div}_{\partial \Omega}, \partial \Omega\right) \rightarrow \boldsymbol{H}^{-1 / 2}\left(\operatorname{curl}_{\partial \Omega}, \partial \Omega\right)$ is an invertible operator only if $k$ is a regular wavenumber (that is, $k^{2}$ is not an eigenvalue of the interior Maxwell eigenvalue problem). Throughout this work, we assume the invertibility of $\mathbf{T}_{\mathrm{e}}$ and hence in (2.13) the set $\mathcal{K}(\Omega)$ of resonant wavenumbers, of the configuration $\Omega$, are excluded from the parameter set $\mathcal{D}$. Henceforth we use $\mathcal{D}$ to denote the parameter set in $\mathbb{R}^{7}$ that does not include $\mathcal{K}(\Omega)$.

Remark 1. The restriction on the resonance wavenumber in the electric field integral equation (EFIE) formulation (2.13) can be avoided by using a combined FIE (CFIE), that combines (2.13) with a relatively simple (weaklysingular) second-kind magnetic FIE (MFIE). In this work, for convenience of presenting the main idea of the multiple scattering reduced basis algorithm, we restrict to the EFIE formulation. However, everything discuss is applicable to the CFIE formulation with minimal modifications.

The mutually adjoint property of the domain and range space of $\mathbf{T}_{\mathrm{e}}$ leads to the variational formulation of (2.13): for $\boldsymbol{\mu} \in \mathcal{D}$, find $\boldsymbol{w}(\boldsymbol{\mu}) \in \boldsymbol{H}^{-1 / 2}\left(\operatorname{div}_{\partial \Omega}, \partial \Omega\right)$ such that

$$
i k Z\left\langle\left(\mathbf{T}_{\mathrm{e}} \boldsymbol{w}\right)(\cdot ; \boldsymbol{\mu}), \boldsymbol{v}\right\rangle=-\left\langle\left(\pi_{T} \boldsymbol{E}^{\mathrm{inc}}\right)(\cdot ; \boldsymbol{\mu}), \boldsymbol{v}\right\rangle \quad \boldsymbol{v} \in \boldsymbol{H}^{-1 / 2}\left(\operatorname{div}_{\partial \Omega}, \partial \Omega\right) .
$$

Thus, reformulation of the scattering process modeled by the exterior Maxwell system through the EFIE and the variational form surface integral equation (2.14) is a natural approach to efficiently compute the electric far field using the representation (2.9). Henceforth our main focus is on the development of an efficient computational approach for solving the parametrized surface integral equation (2.14), and hence fast computation of the RCS, for all $\boldsymbol{\mu} \in \mathcal{D}$, using (2.8)-(2.9).

For any fixed parameter value, the EFIE can subsequently be discretized using a Galerkin approach. Replacing the functional space $\boldsymbol{H}^{-1 / 2}\left(\operatorname{div}_{\partial \Omega}, \partial \Omega\right)$ by some conforming finite dimensional subspace yields the Boundary Element Method (BEM), also known as the Method of Moment (MoM). A common choice as discretization space is the lowest order complex Raviart-Thomas space $\mathbb{R T}_{0}$, often referred to as the Rao-Wilton-Glisson elements in the electromagnetic community.

\section{Boundary element multiple scattering model}

Throughout the paper, for $\boldsymbol{\mu} \in \mathcal{D}, \boldsymbol{w}(\boldsymbol{\mu})=\sum_{\mathrm{j}=1}^{\mathrm{J}} \boldsymbol{w}_{\mathrm{j}}(\boldsymbol{\mu}) \in \boldsymbol{H}^{-1 / 2}\left(\operatorname{div}_{\partial \Omega}, \partial \Omega\right)$, denotes the unique global solution of (2.13), where $\boldsymbol{w}_{\mathrm{i}}(\boldsymbol{\mu})=\left.\boldsymbol{w}\right|_{\partial \Omega_{\mathrm{i}}}(\boldsymbol{\mu})$, for $\mathrm{i}=1, \ldots, \mathrm{J}$. Since

$$
\mathrm{n}(\boldsymbol{x})^{T} \boldsymbol{w}(\boldsymbol{x})=\mathrm{n}(\boldsymbol{x})^{T} \boldsymbol{w}_{\mathrm{i}}(\boldsymbol{x}), \quad \boldsymbol{x} \in \partial \Omega_{\mathrm{i}}, \quad \mathrm{i}=1, \ldots, \mathrm{J},
$$

$\boldsymbol{w} \in \boldsymbol{H}^{-1 / 2}\left(\operatorname{div}_{\partial \Omega}, \partial \Omega\right)$ if and only if $\boldsymbol{w}_{\mathrm{i}} \in \boldsymbol{H}^{-1 / 2}\left(\operatorname{div}_{\partial \Omega_{\mathrm{i}}}, \partial \Omega_{i}\right)$ for $\mathrm{i}=1, \ldots, \mathrm{J}$. Using (2.11), (2.14) and integration by parts, the main task in the electromagnetic scattering problem is: for each $\boldsymbol{\mu} \in \mathcal{D}$, find $\mathrm{J}$ surface currents $\boldsymbol{w}_{\mathrm{j}} \in$ $\boldsymbol{H}^{-1 / 2}\left(\operatorname{div}_{\partial \Omega_{\mathrm{j}}}, \partial \Omega_{\mathrm{j}}\right), \mathrm{j}=1, \ldots, \mathrm{J}$ by solving the coupled surface scattering system

$$
\begin{array}{cc}
\sum_{j=1}^{\mathrm{J}}\left\{a_{1}^{\mathrm{ij}}\left[\boldsymbol{w}_{\mathrm{j}}(\boldsymbol{\mu}), \boldsymbol{v}_{\mathrm{i}} ; \boldsymbol{\mu}\right]+a_{2}^{\mathrm{ij}}\left[\boldsymbol{w}_{\mathrm{j}}(\boldsymbol{\mu}), \boldsymbol{v}_{\mathrm{i}} ; \boldsymbol{\mu}\right]\right\}=f^{\mathrm{i}}\left[\boldsymbol{v}_{\mathrm{i}} ; \boldsymbol{\mu}\right], & \boldsymbol{v}_{\mathrm{i}} \in \boldsymbol{H}^{-1 / 2}\left(\operatorname{div}_{\partial \Omega_{\mathrm{i}}}, \partial \Omega_{\mathrm{i}}\right), \\
& \mathrm{i}=1, \ldots, \mathrm{J},
\end{array}
$$

where $a_{1}^{\mathrm{ij}}[\cdot, \cdot ; \boldsymbol{\mu}], a_{2}^{\mathrm{ij}}[\cdot, \cdot ; \boldsymbol{\mu}]: \boldsymbol{H}^{-1 / 2}\left(\operatorname{div}_{\partial \Omega_{\mathrm{j}}}, \partial \Omega_{\mathrm{j}}\right) \times \boldsymbol{H}^{-1 / 2}\left(\operatorname{div}_{\partial \Omega_{\mathrm{i}}}, \partial \Omega_{\mathrm{i}}\right) \rightarrow \mathbb{C}$ are sesquilinear forms, defined for $\boldsymbol{\psi} \in$ $\boldsymbol{H}^{-1 / 2}\left(\operatorname{div}_{\partial \Omega_{j}}, \partial \Omega_{j}\right), \boldsymbol{\eta} \in \boldsymbol{H}^{-1 / 2}\left(\operatorname{div}_{\partial \Omega_{i}}, \partial \Omega_{\mathrm{i}}\right)$, as

$$
\begin{aligned}
a_{1}^{\mathrm{ij}}[\boldsymbol{\psi}, \boldsymbol{\eta} ; \boldsymbol{\mu}] & =i k Z \int_{\partial \Omega_{\mathrm{i}}} \int_{\partial \Omega_{\mathrm{j}}} \Phi_{k}(\boldsymbol{x}, \boldsymbol{y}) \psi(\boldsymbol{y}) \cdot \overline{\boldsymbol{\eta}(\boldsymbol{x})} d s(\boldsymbol{y}) d s(\boldsymbol{x}), \\
a_{2}^{\mathrm{ij}}[\boldsymbol{\psi}, \boldsymbol{\eta} ; \boldsymbol{\mu}] & =-\frac{i Z}{k} \int_{\partial \Omega_{\mathrm{i}}} \int_{\partial \Omega_{\mathrm{j}}} \Phi_{k}(\boldsymbol{x}, \boldsymbol{y}) \operatorname{div}_{\boldsymbol{y}} \psi(\boldsymbol{y}) \overline{\operatorname{div}_{\boldsymbol{x}} \boldsymbol{\eta}(\boldsymbol{x})} d s(\boldsymbol{y}) d s(\boldsymbol{x}),
\end{aligned}
$$

and

$$
f^{\mathrm{i}}[\boldsymbol{\eta} ; \boldsymbol{\mu}]=-\int_{\partial \Omega_{\mathrm{i}}} \boldsymbol{E}^{\mathrm{inc}}(\boldsymbol{x} ; \boldsymbol{\mu}) \cdot \overline{\boldsymbol{\eta}(\boldsymbol{x})} d s(\boldsymbol{x}) .
$$


Using the unique solution of the system (2.13) and (2.10), we define the J exterior electric fields

$$
\boldsymbol{E}_{\mathrm{i}}(\boldsymbol{x} ; \boldsymbol{\mu})=i k Z\left(\mathbf{S}_{\mathrm{e}} \boldsymbol{w}_{\mathrm{i}}\right)(\boldsymbol{x} ; \boldsymbol{\mu}), \quad \boldsymbol{x} \in \mathbb{R}^{3} \backslash \bar{\Omega}_{i} \quad \mathrm{i}=1, \ldots, \mathrm{J},
$$

such that the exterior field in (2.10) can be written as $\boldsymbol{E}=\sum_{\mathrm{i}=1}^{\mathrm{J}} \boldsymbol{E}_{\mathrm{i}}$. Further, for well separated obstacles [27], for each $\mathrm{i}=1, \ldots, \mathrm{J}, \boldsymbol{E}_{\mathrm{i}}$ is the unique radiating solution of the Maxwell equation exterior to $\bar{\Omega}_{\mathrm{i}}$, that

$$
\operatorname{curl} \operatorname{curl} \boldsymbol{E}_{\mathrm{i}}(\boldsymbol{x} ; \boldsymbol{\mu})-k^{2} \boldsymbol{E}_{i}(\boldsymbol{x} ; \boldsymbol{\mu})=\mathbf{0}, \quad \boldsymbol{x} \in \mathbb{R}^{3} \backslash \bar{\Omega}_{\mathrm{i}},
$$

subject to the reflected wave perfect conductor boundary condition

$$
\left(\pi_{T} \boldsymbol{E}_{i}\right)(\boldsymbol{x} ; \boldsymbol{\mu})=-\left(\pi_{T} \boldsymbol{E}^{\mathrm{inc}}\right)(\boldsymbol{x} ; \boldsymbol{\mu})-\sum_{\substack{j=1 \\ j \neq i}}^{\mathrm{J}}\left(\pi_{T} \boldsymbol{E}_{\mathrm{j}}\right)(\boldsymbol{x} ; \boldsymbol{\mu}), \quad \boldsymbol{x} \in \partial \Omega_{\mathrm{i}} .
$$

The term $\sum_{j=1}^{\mathrm{J}=1}\left(\pi_{T} \boldsymbol{E}_{\mathrm{j}}\right)(\boldsymbol{x} ; \boldsymbol{\mu})$ represents the reflected waves impinging on the surface of the i-th obstacle from all other obstacles in the multiple scatterer configuration. We will later use this physical interpretation to design an efficient online part of the reduced basis algorithm for any parameter $\boldsymbol{\mu} \in \mathcal{D}$, relying a iterative process that mimics this physical picture.

For $i=1, \ldots, \mathrm{J}$, corresponding to the $i$-th scatterer in the configuration $\Omega$, let $\mathbb{V}_{h, \mathrm{i}}$ be an $\mathcal{N}$-dimensional subspace of $\boldsymbol{H}^{0}\left(\operatorname{div}_{\partial \Omega_{\mathrm{i}}}, \partial \Omega_{\mathrm{i}}\right) \subset \boldsymbol{H}^{-1 / 2}\left(\operatorname{div}_{\partial \Omega_{\mathrm{i}}}, \partial \Omega_{\mathrm{i}}\right)$ based on the discretization mesh parameter $h$. In our case we choose the lowest order complex Raviart-Thomas space $\mathbb{V}_{h, \mathrm{i}}=\mathbb{R T}_{0}\left(\partial \Omega_{h, \mathrm{i}}\right)$ spanned, for example, by the Rao-Wilton-Glisson (RWG) basis as an approximation space defined on a mesh $\partial \Omega_{h, \mathrm{i}}$ on the surface $\partial \Omega_{\mathrm{i}}$. Thus, let $\mathbb{V}_{h, \mathrm{i}}=\operatorname{span}\left\{\boldsymbol{\phi}_{\mathrm{n}}^{\mathrm{i}}: \mathrm{n}=1, \ldots, \mathcal{N}\right\}$.

For $\boldsymbol{\mu} \in \mathcal{D}$ and $\mathrm{j}=1, \ldots, \mathrm{J}$, the boundary element Galerkin approximation $\boldsymbol{w}_{h, \mathrm{j}}(\boldsymbol{\mu}) \in \mathbb{V}_{h, \mathrm{j}}=\mathbb{R}_{0}\left(\partial \Omega_{h, j}\right)$ to the unique solution $\boldsymbol{w}_{j}(\boldsymbol{\mu})$ of (3.1) resp. (2.14) is represented as

$$
\boldsymbol{w}_{h, \mathrm{j}}(\boldsymbol{x} ; \boldsymbol{\mu})=\sum_{\mathrm{n}=1}^{\mathcal{N}} \mathrm{w}_{\mathrm{n}}^{\mathrm{j}}(\boldsymbol{\mu}) \boldsymbol{\phi}_{\mathrm{n}}^{\mathrm{j}}(\boldsymbol{x}), \quad \boldsymbol{x} \in \partial \Omega_{\mathrm{j}}, \quad \mathrm{j}=1, \ldots, \mathrm{J} .
$$

Note that the accuracy of the boundary element Galerkin approximation $\boldsymbol{w}_{h, j}(\boldsymbol{\mu})$ depends on $\mathcal{N}$ as well as a number of physical variables, in particular the wave-number $k$. We therefore choose $\mathcal{N}$ to be large enough to ensure that the surface currents $\boldsymbol{w}_{h, \mathrm{j}}(\boldsymbol{\mu})$ are accurately enough represented for all wave-numbers $k \in\left[k^{-}, k^{+}\right]$.

Using (3.1), for each fixed $\boldsymbol{\mu} \in \mathcal{D}$, and $\mathrm{j}=1, \ldots, \mathrm{J}$, the boundary element Galerkin approximation $\boldsymbol{w}_{h, \mathrm{j}}(\boldsymbol{\mu}) \in \mathbb{V}_{h, \mathrm{j}}$ is sought as solution of the discrete variational problem

$$
\sum_{j=1}^{\mathrm{J}} a^{\mathrm{ij}}\left[\boldsymbol{w}_{h, \mathrm{j}}(\boldsymbol{\mu}), \boldsymbol{v}_{h, \mathrm{j}} ; \boldsymbol{\mu}\right]=f^{\mathrm{i}}\left[\boldsymbol{v}_{h, \mathrm{j}} ; \boldsymbol{\mu}\right], \quad \forall \boldsymbol{v}_{h, \mathrm{j}} \in \mathbb{V}_{h, \mathrm{i}}, \quad \mathrm{i}=1, \ldots, \mathrm{J},
$$

where, $a^{\mathrm{ij}}[\cdot, \cdot ; \boldsymbol{\mu}]: \boldsymbol{H}^{-1 / 2}\left(\operatorname{div}_{\partial \Omega_{\mathrm{j}}}, \partial \Omega_{\mathrm{j}}\right) \times \boldsymbol{H}^{-1 / 2}\left(\operatorname{div}_{\partial \Omega_{\mathrm{i}}}, \partial \Omega_{\mathrm{i}}\right) \rightarrow \mathbb{C}$ is defined as

$$
a^{i j}[\cdot, \cdot ; \boldsymbol{\mu}]=a_{1}^{i j}[\cdot, \cdot ; \boldsymbol{\mu}]+a_{2}^{\mathrm{ij}}[\cdot, \cdot ; \boldsymbol{\mu}]
$$

Remark 2. For notational convenience we refer to the solution of (3.9) as the truth solution. This is meant to indicate that $\mathcal{N}$ is assumed to be large enough to guarantee that the solution is accurate to within a user specific error tolerance. In other words, we take this solution to be as close the exact solution as required to for the application of interest.

Remark 3. The above framework can also be applied for the case of a single scatterer $\Omega$. The problem then reads: for each fixed $\boldsymbol{\mu} \in \mathcal{D}$, find $\boldsymbol{w}_{h}(\boldsymbol{\mu}) \in \mathbb{R}_{0}\left(\partial \Omega_{h}\right)$ such that

$$
a\left[\boldsymbol{w}_{h}(\boldsymbol{\mu}), \boldsymbol{v}_{h} ; \boldsymbol{\mu}\right]=f\left[\boldsymbol{v}_{h} ; \boldsymbol{\mu}\right], \quad \forall \boldsymbol{v}_{h} \in \mathbb{R}_{0}\left(\partial \Omega_{h}\right),
$$

where $a\left[\boldsymbol{w}_{h}(\boldsymbol{\mu}), \boldsymbol{v}_{h} ; \boldsymbol{\mu}\right]:=a^{11}\left[\boldsymbol{w}_{h}(\boldsymbol{\mu}), \boldsymbol{v}_{h} ; \boldsymbol{\mu}\right]$ and $f\left[\boldsymbol{v}_{h} ; \boldsymbol{\mu}\right]:=f^{1}\left[\boldsymbol{v}_{h} ; \boldsymbol{\mu}\right]$ in combination with $\Omega_{1}=\Omega$. 
Further, note that the matrix representation of (3.9) is given for each $\mu \in \mathcal{D}$ and $i, j=1, \ldots, \mathrm{J}$ by

$$
\begin{aligned}
{\left[\mathrm{M}^{\mathrm{ij}}(\boldsymbol{\mu})\right]_{m, n}=a^{\mathrm{ij}}\left[\boldsymbol{\phi}_{n}^{\mathrm{i}}, \boldsymbol{\phi}_{m}^{\mathrm{i}} ; \boldsymbol{\mu}\right], \quad\left[\mathrm{f}^{\mathrm{i}}(\boldsymbol{\mu})\right]_{m}=f^{\mathrm{i}}\left[\boldsymbol{\phi}_{m}^{\mathrm{i}} ; \boldsymbol{\mu}\right], \quad\left[\mathrm{w}^{\mathrm{i}}(\boldsymbol{\mu})\right]_{n}=\mathrm{w}_{n}^{i}(\boldsymbol{\mu}) } \\
m=1, \ldots, \mathcal{N}, \quad n=1, \ldots, \mathcal{N} .
\end{aligned}
$$

The unique solution of (3.9), for each $\mu \in \mathcal{D}$, can (at least in theory) be computed by solving the global $\mathrm{JN} \times \mathrm{JN}$ linear system:

$$
\sum_{j=1}^{\mathrm{J}} \mathrm{M}^{\mathrm{ij}}(\boldsymbol{\mu}) \mathrm{w}^{\mathrm{j}}(\boldsymbol{\mu})=\mathrm{f}^{\mathrm{i}}(P), \quad \mathrm{i}=1, \ldots, \mathrm{J} .
$$

The above approach is prohibitively expensive if the electromagnetic scattering problem needs to be solved for many times to model parametric variation across $\boldsymbol{\mu} \in \mathcal{D}$. Indeed, even for a single fixed parameter $\boldsymbol{\mu}$, this approach is not practically useful if $\mathrm{J}$ is large since the dimension $\mathcal{N}$ of the each of the $\mathrm{J}$ boundary element spaces can be large (even for $1 \%$ accuracy) and that $\mathrm{M}^{i j}$ are dense complex matrices. We may avoid solving the coupled global system by some iterative procedure [23] based on the observation in (3.5)-(3.6), but this approach also requires solving J uncoupled $\mathcal{N} \times \mathcal{N}$ system of equations at each iteration. The large number of degrees of freedom of the boundary element discretization for the three dimensional scattering problem likewise makes this expensive.

\subsection{Boundary element model described on reference shapes}

In this section, we reformulate problem (3.9) for particular geometric configurations that arise from the mapping of reference shapes into the physical space using affine transformations. For sake of simplicity, we assume that each obstacle $\Omega_{\mathrm{j}}$ is the image of an affine transformation $T_{\mathrm{j}}$ from only one reference shape $\widehat{D}$ onto $\Omega_{\mathrm{j}}$, for each $\mathrm{j}=1, \ldots, \mathrm{J}$. In particular, we assume that $T_{\mathrm{j}}: \widehat{D} \rightarrow \Omega_{\mathrm{j}}$ is of the form $T_{\mathrm{j}}(\hat{\boldsymbol{x}})=\gamma_{\mathrm{j}} \mathrm{B}_{\mathrm{j}} \hat{\boldsymbol{x}}+\boldsymbol{b}_{\mathrm{j}}$ where $\gamma_{\mathrm{j}} \in \mathbb{R}$ is a stretching/shrinking factor, $\mathrm{B}_{\mathrm{j}} \in \mathbb{R}^{3 \times 3}$ a rotation matrix and $\boldsymbol{b}_{\mathrm{j}} \in \mathbb{R}^{3}$ a translation vector. As we will see in Section 6 , the assumption of just involving one type of reference element is not essential and different types of scatterers can be considered.

For $\mathrm{j}=1, \ldots, \mathrm{J}$, let $\widehat{\mathcal{P}}_{\mathrm{j}}: \boldsymbol{H}^{-1 / 2}\left(\operatorname{div}_{\partial \Omega_{\mathrm{j}}}, \partial \Omega_{\mathrm{j}}\right) \rightarrow \boldsymbol{H}^{-1 / 2}\left(\operatorname{div}_{\partial \widehat{D}}, \partial \widehat{D}\right)$ denote the Piola transformation from the physical surface $\partial \Omega_{\mathrm{j}}$ to the reference surface $\partial \widehat{D}$, defined as

$$
\left(\widehat{\mathcal{P}}_{\mathrm{i}} \psi\right)(\hat{\boldsymbol{x}})=\mathrm{B}_{\mathrm{j}}^{T} \psi\left(T_{\mathrm{j}}(\hat{\boldsymbol{x}})\right), \quad \boldsymbol{\psi} \in \boldsymbol{H}^{-1 / 2}\left(\operatorname{div}_{\partial \Omega_{\mathrm{j}}}, \partial \Omega_{\mathrm{j}}\right) \quad \hat{\boldsymbol{x}} \in \partial \widehat{D}
$$

where $T_{\mathrm{j}} \hat{\boldsymbol{x}}=\gamma_{\mathrm{j}} \mathrm{B}_{\mathrm{j}} \hat{\boldsymbol{x}}+\boldsymbol{b}_{\mathrm{j}}$, with $\mathrm{B}_{\mathrm{j}}^{T}=\mathrm{B}_{\mathrm{j}}^{-1}$. To show some properties of the Piola transformation, observe that the normal resp. tangential vectors on $\partial \widehat{D}$ and $\partial \Omega_{j}$ are linked through the following relation

$$
\mathrm{B}_{\mathrm{j}} \hat{\mathrm{n}}(\hat{\boldsymbol{x}})=\mathrm{n}\left(T_{\mathrm{j}}(\hat{\boldsymbol{x}})\right) \quad \text { and } \quad \mathrm{B}_{\mathrm{j}} \hat{\boldsymbol{v}}(\hat{\boldsymbol{x}})=\boldsymbol{v}\left(T_{\mathrm{j}}(\hat{\boldsymbol{x}})\right),
$$

where $\mathrm{n}$ is the normal on $\partial \Omega_{\mathrm{j}}$, $\hat{\mathrm{n}}$ the normal on $\partial \widehat{D}$ and $v$ resp. $\hat{v}$ any one to one mapping of a tangential vector. The Piola transformation conserves tangential functions: if $\psi(x) \cdot n(x)=0$ on $\partial \Omega_{j}$, then

$$
\left(\widehat{\mathcal{P}}_{\mathrm{j}} \psi\right)(\hat{\boldsymbol{x}}) \cdot \hat{\mathrm{n}}(\hat{\boldsymbol{x}})=\left[\mathrm{B}_{\mathrm{j}}^{T} \psi\left(T_{\mathrm{j}}(\hat{\boldsymbol{x}})\right)\right] \cdot\left[\mathrm{B}_{\mathrm{j}}^{T} \mathrm{n}\left(T_{\mathrm{j}}(\hat{\boldsymbol{x}})\right)\right]=\boldsymbol{\psi}\left(T_{\mathrm{j}}(\hat{\boldsymbol{x}})\right) \cdot \mathrm{n}\left(T_{\mathrm{j}}(\hat{\boldsymbol{x}})\right)=0,
$$

since $\mathrm{B}_{\mathrm{j}}$ is a rotation matrix and $T_{\mathrm{j}}(\hat{\boldsymbol{x}}) \in \partial \Omega_{\mathrm{j}}$.

Let us further be precise on how we build the boundary element truth approximation space $\mathbb{V}_{h, \mathrm{i}}$. Given a mesh $\partial \widehat{D}_{h}$ on the reference shape $\partial \widehat{D}$, we use the affine transformation $T_{\mathrm{i}}$ to construct a mesh $\partial \Omega_{\mathrm{i}, h}$ on the surface $\partial \Omega_{\mathrm{i}}$. As an approximation space on $\partial \Omega_{i}$ we then choose, as already mentioned above, the lowest order complex Raviart-Thomas space $\mathbb{R T}_{0}\left(\partial \Omega_{\mathrm{i}, h}\right)$.

The degrees of freedom of $\mathbb{R T}_{0}\left(\partial \widehat{D}_{h}\right)$ resp. $\mathbb{R T}_{0}\left(\partial \Omega_{\mathrm{i}, h}\right)$ are conserved by the Piola transformation: For the lowest order Raviart-Thomas space, edge fluxes are the appropriate degrees of freedom. Let $\hat{e}$ be any edge of the mesh of the surface $\partial \widehat{D}_{h}$ of the element $\widehat{E}$, i.e. $\hat{e} \subset \partial \widehat{E}$. Let $e$ and $E$ be the corresponding edge and element on the physical mesh $\partial \Omega_{\mathrm{i}, h}$. Let $\hat{\boldsymbol{v}}_{\hat{e}}$ and $\boldsymbol{v}_{e}$ be the outer normal of the element $\widehat{E}$ resp. $E$ on the edge $\hat{e}$ resp. $e$. In addition, let $\widehat{\boldsymbol{\phi}}_{\hat{f}}$ and $\boldsymbol{\phi}_{f}$ be the basis functions of $R T_{0}\left(\partial \widehat{D}_{h}\right)$ resp. $R T_{0}\left(\partial \Omega_{\mathrm{i}, h}\right)$ associated to the edge $\hat{f}$ resp. $f$ satisfying $\widehat{\boldsymbol{\phi}}_{\hat{f}}=\widehat{\mathcal{P}}_{\mathrm{i}} \boldsymbol{\phi}_{f}$. Then, $\boldsymbol{\phi}_{f}$ satisfies

$$
\int_{e} \phi_{f}(x) \cdot v_{e}(x)=\delta_{e f}
$$


and hence using integration by substitution with the affine mapping $T_{\mathrm{i}}$ from $\hat{e}$ to $e$, we have

$$
\begin{aligned}
\int_{\hat{e}} \widehat{\boldsymbol{\phi}}_{\hat{f}}(\hat{\boldsymbol{x}}) \cdot \boldsymbol{v}_{\hat{e}}(\hat{\boldsymbol{x}}) & =\gamma_{\mathrm{i}} \int_{\hat{e}} \mathrm{~B}_{\mathrm{i}}^{T} \boldsymbol{\phi}_{f}\left(T_{\mathrm{i}}(\hat{\boldsymbol{x}})\right) \cdot \mathrm{B}_{\mathrm{i}}^{T} \boldsymbol{v}_{e}\left(T_{\mathrm{i}}(\hat{\boldsymbol{x}})\right) \\
& =\gamma_{\mathrm{i}} \int_{\hat{e}} \boldsymbol{\phi}_{f}\left(T_{\mathrm{j}}(\hat{\boldsymbol{x}})\right) \cdot \boldsymbol{v}_{e}\left(T_{\mathrm{i}}(\hat{\boldsymbol{x}})\right)=\int_{e} \boldsymbol{\phi}_{f}(\boldsymbol{x}) \cdot \boldsymbol{v}_{e}(\boldsymbol{x})=\delta_{e f}=\delta_{\hat{e} \hat{f}} .
\end{aligned}
$$

Thus the dimension of the boundary element space for the $i$-th obstacle in the configuration is $\mathcal{N}$ (independent of $i$ ), the dimension of $\mathbb{R} \mathbb{T}_{0}\left(\partial \widehat{D}_{h}\right)$.

Since the Piola transform $\widehat{\mathcal{P}}_{j}$ is an isomorphism between $\boldsymbol{H}^{0}\left(\operatorname{div}_{\partial \Omega_{\mathrm{j}}}, \partial \Omega_{\mathrm{j}}\right)$ and $\boldsymbol{H}^{0}\left(\operatorname{div}_{\partial \widehat{D}}, \partial \widehat{D}\right)$, we can reformulate problem (3.9) as a problem with the computational domain $\partial \widehat{D}$ only.

To do so we first map the sesquilinear forms in (3.2)-(3.3) to the reference surface $\partial \widehat{D}$. Let $\boldsymbol{\psi} \in \boldsymbol{H}^{-1 / 2}\left(\operatorname{div}_{\partial \Omega_{\mathrm{j}}}, \partial \Omega_{\mathrm{j}}\right)$, $\boldsymbol{\eta} \in \boldsymbol{H}^{-1 / 2}\left(\operatorname{div}_{\partial \Omega_{\mathrm{i}}}, \partial \Omega_{\mathrm{i}}\right), \mathrm{i}, \mathrm{j}=1, \ldots, \mathrm{J}$. Using (3.2) and integration by substitution with $\boldsymbol{x}=T_{\mathrm{i}}(\hat{\boldsymbol{x}})$ and $\boldsymbol{y}=T_{\mathrm{j}}(\hat{\boldsymbol{y}})$ yields

$$
a_{1}^{\mathrm{ij}}[\boldsymbol{\psi}, \boldsymbol{\eta} ; \boldsymbol{\mu}]=i k Z \gamma_{\mathrm{i}} \gamma_{\mathrm{j}} \int_{\partial \widehat{D}} \int_{\partial \widehat{D}} \widehat{\Phi}_{k}^{\mathrm{ij}}(\hat{\boldsymbol{x}}, \hat{\boldsymbol{y}})\left(\mathrm{B}_{\mathrm{j}} \widehat{\boldsymbol{\psi}}_{\mathrm{j}}(\hat{\boldsymbol{y}})\right) \cdot\left(\mathrm{B}_{\mathrm{i}} \overline{\widehat{\boldsymbol{\eta}}_{\mathrm{i}}(\hat{\boldsymbol{x}})}\right), d s(\hat{\boldsymbol{y}}) d s(\hat{\boldsymbol{x}}) .
$$

where,

$$
\widehat{\Phi}_{k}^{\mathrm{ij}}(\hat{\boldsymbol{x}}, \hat{\boldsymbol{y}})=\Phi_{k}\left(T_{\mathrm{i}}(\hat{\boldsymbol{x}}), T_{\mathrm{j}}(\hat{\boldsymbol{y}})\right), \quad \widehat{\boldsymbol{\psi}}_{\mathrm{j}}(\hat{\boldsymbol{y}})=\left(\widehat{\mathcal{P}}_{\mathrm{j}} \psi\right)(\hat{\boldsymbol{y}}), \quad \widehat{\boldsymbol{\eta}}_{\mathrm{i}}(\hat{\boldsymbol{x}})=\left(\widehat{\mathcal{P}}_{\mathrm{i}} \boldsymbol{\eta}\right)(\hat{\boldsymbol{x}}), \quad \hat{\boldsymbol{x}}, \hat{\boldsymbol{y}} \in \partial \widehat{D} .
$$

Similarly, using (3.3) we get

$$
a_{2}^{\mathrm{ij}}[\boldsymbol{\psi}, \boldsymbol{\eta} ; \boldsymbol{\mu}]=-\frac{i Z \gamma_{\mathrm{i}} \gamma_{\mathrm{j}}}{k} \int_{\partial \widehat{D}} \int_{\partial \widehat{D}} \widehat{\Phi}_{k}^{\mathrm{ij}}(\hat{\boldsymbol{x}}, \hat{\boldsymbol{y}}) \operatorname{div}_{\boldsymbol{y}} \boldsymbol{\psi}\left(T_{\mathrm{j}}(\hat{\boldsymbol{y}})\right) \overline{\operatorname{div}_{\boldsymbol{x}} \boldsymbol{\eta}\left(T_{\mathrm{i}}(\hat{\boldsymbol{x}})\right)} d s(\hat{\boldsymbol{y}}) d s(\hat{\boldsymbol{x}}),
$$

Observe that $\nabla_{y}=\gamma_{j}^{-1} B_{j} \nabla_{\hat{y}}$ and thus the surface divergence can be written as

$$
\begin{aligned}
\operatorname{div}_{y} \psi\left(T_{j}(\hat{\boldsymbol{y}})\right) & =\left[\nabla_{\boldsymbol{y}}-\mathrm{n}\left(T_{\mathrm{j}}(\hat{\boldsymbol{y}})\right) \mathrm{n}\left(T_{\mathrm{j}}(\hat{\boldsymbol{y}})\right) \cdot \nabla_{\boldsymbol{y}}\right] \cdot \boldsymbol{\psi}\left(T_{\mathrm{j}}(\hat{\boldsymbol{y}})\right) \\
& =\gamma_{j}^{-1}\left[\mathrm{~B}_{j} \nabla_{\hat{\boldsymbol{y}}}-\mathrm{B}_{\mathrm{j}} \widehat{\mathrm{n}}(\hat{\boldsymbol{y}}) \mathrm{B}_{\mathrm{j}} \widehat{\mathrm{n}}(\hat{\boldsymbol{y}}) \cdot \mathrm{B}_{j} \nabla_{\hat{y}}\right] \cdot \mathrm{B}_{j} \widehat{\boldsymbol{\psi}}_{\mathrm{j}}(\hat{\boldsymbol{y}}) \\
& =\gamma_{j}^{-1}\left[\nabla_{\hat{\boldsymbol{y}}}-\hat{\mathrm{n}}(\hat{\boldsymbol{y}}) \hat{\mathrm{n}}(\hat{\boldsymbol{y}}) \cdot \nabla_{\hat{y}}\right] \cdot \widehat{\boldsymbol{\psi}}_{\mathrm{j}}(\hat{\boldsymbol{y}}) \\
& =\gamma_{j}^{-1} \operatorname{div}_{\hat{\boldsymbol{y}}} \widehat{\boldsymbol{\psi}}_{\mathrm{j}}(\hat{\boldsymbol{y}}) .
\end{aligned}
$$

Using (3.15)-(3.18) in (3.10),

$$
a^{\mathrm{ij}}[\boldsymbol{\psi}, \boldsymbol{\eta} ; \boldsymbol{\mu}]=\widehat{a}^{\mathrm{ij}}\left[\widehat{\boldsymbol{\psi}}_{\mathrm{j}}, \widehat{\boldsymbol{\eta}}_{\mathrm{i}} ; \boldsymbol{\mu}\right],
$$

where for $\widehat{\boldsymbol{\psi}}, \widehat{\boldsymbol{\eta}} \in \boldsymbol{H}^{-1 / 2}\left(\operatorname{div}_{\partial \widehat{D}}, \partial \widehat{D}\right)$,

$$
\widehat{a}^{\mathrm{ij}}[\widehat{\boldsymbol{\psi}}, \widehat{\boldsymbol{\eta}} ; \boldsymbol{\mu}]=i k Z \gamma_{\mathrm{i}} \gamma_{\mathrm{j}} \int_{\partial \widehat{D}} \int_{\partial \widehat{D}} \widehat{\Phi}_{k}^{\mathrm{ij}}(\hat{\boldsymbol{x}}, \hat{\boldsymbol{y}})\left(\left(\mathrm{B}_{j} \widehat{\boldsymbol{\psi}}(\hat{\boldsymbol{y}})\right) \cdot\left(\mathrm{B}_{\mathrm{i}} \overline{\overline{\boldsymbol{\eta}}(\hat{\boldsymbol{x}}))}-\frac{1}{k^{2} \gamma_{\mathrm{i}} \gamma_{\mathrm{j}}} \operatorname{div}_{\hat{\boldsymbol{y}}} \widehat{\boldsymbol{\psi}}(\hat{\boldsymbol{y}}) \operatorname{div}_{\hat{\boldsymbol{x}}} \overline{\overline{\boldsymbol{\eta}}(\hat{\boldsymbol{x}})}\right) d s(\hat{\boldsymbol{y}}) d s(\hat{\boldsymbol{x}})\right.
$$

Similarly, using (3.4) and $T_{\mathrm{j}}: \partial \widehat{D} \rightarrow \partial \Omega_{j}$, the input source function in (3.1) can be written, for $\mathrm{i}=1, \ldots, \mathrm{J}$, as

$$
f^{\mathrm{i}}[\boldsymbol{\eta} ; \boldsymbol{\mu}]=\hat{f}^{\mathrm{i}}\left[\widehat{\boldsymbol{\eta}}_{\mathrm{i}} ; \boldsymbol{\mu}\right],
$$

where for $\widehat{\boldsymbol{\eta}} \in \boldsymbol{H}^{-1 / 2}\left(\operatorname{div}_{\partial \widehat{D}}, \partial \widehat{D}\right)$

$$
\hat{f}^{\mathrm{i}}[\widehat{\boldsymbol{\eta}} ; \boldsymbol{\mu}]=-\gamma_{\mathrm{i}} \int_{\partial \widehat{D}} \mathrm{~B}_{\mathrm{i}}^{T} \boldsymbol{E}^{\mathrm{inc}}\left(T_{\mathrm{i}}(\hat{\boldsymbol{x}}) ; \boldsymbol{\mu}\right) \cdot \overline{\overline{\boldsymbol{\eta}}(\hat{\boldsymbol{x}})} d s(\hat{\boldsymbol{x}}) .
$$

We therefore denote $\widehat{\mathbb{V}}_{h}=\mathbb{R T}_{0}\left(\partial \widehat{D}_{h}\right)$ and reformulate the truth approximation as: for each fixed $\mu \in \mathcal{D}$, and $\mathrm{j}=$ $1, \ldots, \mathrm{J}$, find $\widehat{\boldsymbol{w}}_{h, \mathrm{j}}(\boldsymbol{\mu}) \in \widehat{\mathbb{V}}_{h}$ such that

$$
\left.\sum_{j=1}^{\mathrm{J}} \widehat{a}^{\mathrm{ij}}\left[\widehat{\boldsymbol{w}}_{h, \mathrm{j}}(\boldsymbol{\mu}), \widehat{\boldsymbol{v}}_{h} ; \boldsymbol{\mu}\right]=\hat{f}^{\mathrm{i}} \widehat{\boldsymbol{v}}_{h} ; \boldsymbol{\mu}\right], \quad \forall \widehat{\boldsymbol{v}}_{h} \in \widehat{\mathbb{V}}_{h}, \quad \mathrm{i}=1, \ldots, \mathrm{J} .
$$


The physical unknown $\boldsymbol{w}_{h, \mathrm{j}}(\boldsymbol{\mu})$ is then obtained using the inverse $\widehat{\mathcal{P}}_{\mathrm{j}}^{-1}$ of the Piola transformation, i.e., $\boldsymbol{w}_{h, \mathrm{j}}(\boldsymbol{\mu})=$ $\widehat{\mathcal{P}}_{\mathrm{j}}^{-1} \widehat{\boldsymbol{w}}_{h, \mathrm{j}}(\boldsymbol{\mu})$. This reformulation of the underlying boundary element problem will be fundamental in the upcoming design of the reduced basis method.

\section{The Reduced Basis Method}

We consider the parametrized multiple scattering model introduced in Section 3. The parameters of this model consist of the wavenumber, the angle of the incident plane wave, it's polarization and the geometrical configuration of the different scatterers. The goal of this section is to construct a model for this parametrized problem that results in an input-output procedure where the inputs are the parameter values and the output consists of the corresponding radar signature (RCS). This model should respond accurately and be efficient, where we define efficiency in this context to mean that the complexity of the input-output procedure is independent of the dimension of the boundary element space.

We continue to make the assumption that all obstacles $\Omega_{i}$ are images of the reference geometry $\hat{D}$ under an affine transformation $T_{\mathrm{i}}$. The framework that we present is nevertheless general enough so that it can be extended to cases where several reference shapes are considered. However, this substantially increases technical details, notation etc and we limit the discussion here to simply demonstrate the ability to do so in the examples in Section 6.

The overall strategy of the Reduced Basis Method in context of multiple obstacles reads as follows:

1. Assemble a reduced basis $\widehat{\mathbb{V}}_{N}$ for a reference geometry $\hat{D}$ that is trained to respond accurately for all angles of the incident plane wave, its polarization and wave numbers in a certain region $\left[k^{-}, k^{+}\right] \backslash \mathcal{K}(\hat{D})$.

2. Using the affine transformation $T_{i}$, map the reduced basis $\widehat{\mathbb{V}}_{N}$ onto the physical obstacles $D_{\mathrm{i}}$ where they are established as solution spaces $\mathbb{V}_{N, i}$. In view of Section 3.1, we can solve the globally coupled problem directly on the reference shape using the solution space $\widehat{\mathbb{V}}_{N}$.

3. Using an iterative scheme based on the operators acting on the reduced basis $\mathbb{V}_{N, \mathrm{i}}$ (instead on $\mathbb{V}_{h, \mathrm{i}}$ ), we solve the multi-object scattering problem in an iterative manner.

We seek to combine the advantages of an iterative physically intuitive scheme and a model reduction. The fundamental idea behind this approach is that the reflected wave from one scatterer to the other can be approximated by a linear combination of plane waves. The expected limitations of this approach is of course when two obstacle are close to each other. In this case the reflection waves can no more be accurately approached by a linear combination of plane wave, and the approach will fail to produce accurate results.

Before we introduce the reduced model for the coupled system, let us first introduce the reduced basis method for a single obstacle as this will be needed for the above stated point 1 .

\subsection{The Reduced Basis Method for single obstacle scattering problems}

Let us restrict our attention to the case of one single scatterer $\widehat{D}$, in this case the reference obstacle. We refer to [22] for a detailed discussion of the Reduced Basis Method for single obstacle problems. As introduced in Section 3 the parameters are denoted by $\boldsymbol{\mu}=\left(k, \widetilde{\boldsymbol{d}}_{\text {inc }}, \boldsymbol{p}_{\text {inc }}\right) \in \mathcal{D} \subset \mathbb{R}^{7}$. Since we are only dealing with a single scatterer we drop the index $i$, used to indicate the index of the scatterer. The first step of the reduced basis method consists then of providing a subspace $\widehat{\mathbb{V}}_{N}$ of the boundary element space $\widehat{\mathbb{V}}_{h}=\mathbb{R} \mathbb{T}_{0}\left(\partial \widehat{D}_{h}\right)$ that is an approximation of the parametrized solution space

$$
\mathcal{M}_{h}=\left\{\boldsymbol{w}_{h}(\boldsymbol{\mu}) \mid \forall \boldsymbol{\mu} \in \mathcal{D}\right\},
$$

where the Galerkin solutions $\boldsymbol{w}_{h}(\boldsymbol{\mu}) \in \widehat{\mathbb{V}}_{h}$ are the solutions of (3.11) with $\Omega=\widehat{D}$.

For a given reduced basis space $\widehat{\mathbb{V}}_{N} \subset \widehat{\mathbb{V}}_{h}$, the reduced basis approximation solves: for a fixed $\boldsymbol{\mu} \in \mathcal{D}$, find $\boldsymbol{w}_{N}(\boldsymbol{\mu}) \in \mathbb{V}_{N}$ such that

$$
a\left[\boldsymbol{w}_{N}(\boldsymbol{\mu}), \boldsymbol{\xi}_{n} ; \boldsymbol{\mu}\right]=f\left[\boldsymbol{\xi}_{n} ; \boldsymbol{\mu}\right], \quad \forall n=1, \ldots, N,
$$

where $\left\{\boldsymbol{\xi}_{n}\right\}_{n=1}^{N}$ is a basis of $\widehat{\mathbb{V}}_{N}$. More precisely, the reduced basis space $\widehat{\mathbb{V}}_{N}$ is the span of particular "snapshots", i.e.

$$
\widehat{\mathbb{V}}_{N}=\operatorname{span}\left\{\boldsymbol{w}_{h}\left(\boldsymbol{\mu}_{n}\right) \mid n=1, \ldots, N\right\}
$$


The choice of the sample points $\left\{\boldsymbol{\mu}_{n}\right\}_{n=1}^{N}$ is based on a greedy selection over the parameter domain and involves a residual based a posteriori error estimation of the error between the reduced basis approximation $\boldsymbol{w}_{N}(\boldsymbol{\mu})$ and the "truth" approximation $\boldsymbol{w}_{h}(\boldsymbol{\mu})$. Additionally, a Gram-Schmidt orthonormalization is used to obtain a orthonormal basis $\left\{\boldsymbol{\xi}_{n}\right\}_{n=1}^{N}$ for $\widehat{\mathbb{V}}_{N}$. The greedy algorithm requires only $N$ times solving a full boundary element computation (3.11). We refer to [22] for more details.

With the reduced basis approximation (4.1) now being defined, we focus on the fast/efficient implementation of the solution of (4.1). If the corresponding linear system is solved as stated, the assembling time of the matrix and right hand side depends on the dimension $\mathcal{N}$ of the boundary element space $\widehat{\mathbb{V}}_{h}$. To overcome this, let us furthermore assume that the sesquilinear form $a[\cdot, \cdot ; \boldsymbol{\mu}]$ and the linear form $f[\cdot ; \boldsymbol{\mu}]$ is expressed on through the following affine decomposition:

$$
\begin{gathered}
a[\boldsymbol{w}, \boldsymbol{v} ; \boldsymbol{\mu}]=\sum_{q=1}^{Q_{\mathrm{a}}} \theta_{\mathrm{a}, q}(\boldsymbol{\mu}) a_{q}[\boldsymbol{w}, \boldsymbol{v}], \\
f[\boldsymbol{v} ; \boldsymbol{\mu}]=\sum_{q=1}^{Q_{\mathrm{f}}} \theta_{\mathrm{f}, q}(\boldsymbol{\mu}) f_{q}[\boldsymbol{v}],
\end{gathered}
$$

for all $\boldsymbol{w}, \boldsymbol{v} \in \widehat{\mathbb{V}}_{h}$.

Satisfying this affine assumption brings the following advantage: Once the reduced basis $\widehat{\mathbb{V}}_{N}$ is known, the matrices $\mathrm{A}_{q}$ and vectors $\mathrm{f}_{q}$ corresponding to the parameter independent forms $a_{q}$ and $f_{q}$ can be precomputed once and for all. Observe that the matrices $\mathrm{A}^{q}$ are of size $N \times N$ and the vectors $\mathrm{f}_{q}$ of size $N$ independent of $\mu$. For any new parameter value $\boldsymbol{\mu}$ the matrix $\mathrm{A}_{\boldsymbol{\mu}}$ and vector $\mathrm{f}_{\boldsymbol{\mu}}$ corresponding to the forms $a[\cdot, \cdot ; \boldsymbol{\mu}]$ and $f[\cdot ; \boldsymbol{\mu}]$ can be assembled independently on the dimension $\mathcal{N}$ of the boundary element space $\widehat{\mathbb{V}}_{h}$, i.e.,

$$
\mathrm{A}_{\boldsymbol{\mu}}=\sum_{q=1}^{Q_{\mathrm{a}}} \theta_{\mathrm{a}, q}(\boldsymbol{\mu}) \mathrm{A}_{q}, \quad \text { and } \quad \mathrm{f}_{\boldsymbol{\mu}}=\sum_{q=1}^{Q_{\mathrm{f}}} \theta_{\mathrm{f}, q}(\boldsymbol{\mu}) \mathrm{f}_{q} .
$$

Then, the reduced basis approximation is obtained by solving the linear system

$$
\mathrm{A}_{\mu} \mathrm{w}_{\mu}=\mathrm{f}_{\mu}
$$

and writing $\boldsymbol{w}_{N}(\boldsymbol{x}, \boldsymbol{\mu})=\sum_{n=1}^{N} \mathrm{w}(\boldsymbol{\mu})_{n} \boldsymbol{\xi}_{n}(\boldsymbol{x})$. The whole process of assembling the matrix, right hand side and solving the linear system is independent of the dimension $\mathcal{N}$ of the boundary element space $\widehat{\mathbb{V}}_{h}$.

We observe that the affine assumption fails in the case the EFIE this decomposition

$$
\begin{aligned}
a[\boldsymbol{w}, \boldsymbol{v} ; \boldsymbol{\mu}] & =i k Z \int_{\partial \widehat{D}} \int_{\partial \widehat{D}} \Phi_{k}(\boldsymbol{x}, \boldsymbol{y})\left(\boldsymbol{w}(\boldsymbol{y}) \cdot \overline{\boldsymbol{v}(\boldsymbol{x})}-\frac{1}{k^{2}} \operatorname{div} \boldsymbol{w}(\boldsymbol{y}) \overline{\operatorname{div} \boldsymbol{v}(\boldsymbol{x})}\right) d s(\boldsymbol{y}) d s(\boldsymbol{x}), \\
f[\boldsymbol{v} ; \boldsymbol{\mu}] & =-\int_{\partial \widehat{D}} \boldsymbol{E}^{\mathrm{inc}}(\boldsymbol{x} ; \boldsymbol{\mu}) \cdot \overline{\boldsymbol{v}(\boldsymbol{x})} d s(\boldsymbol{x}),
\end{aligned}
$$

due to the presence of the kernel function resp. the nature of the incident field. Since this is at the very core of the efficiency of the method, we will return to this particular issue in more detail in Section 5. For now, we ask the reader to simply accept that this can be overcome to an desired accuracy.

To summarize, the method consists of two main parts, the Offline- and the Online-part. During the Offline part, we spend some time exploring the solution space $\mathcal{M}_{h}$ empirically. Once this task is completed we precompute the $N \times N$-dimensional matrices $\mathrm{A}_{q}$ and the $N$-dimensional vectors $\mathrm{f}_{q}$. During the Online-part, where we expect to evaluate the solution for many parameter values, for each new parameter value $\boldsymbol{\mu}$ we determine the scalar coefficients $\theta_{\mathrm{a}, q}(\boldsymbol{\mu})$, $\theta_{\mathrm{f}, q}(\boldsymbol{\mu})$ before we build the matrix $\mathrm{A}_{\boldsymbol{\mu}}$ and the vector $\mathrm{f}_{\boldsymbol{\mu}}$ and solve the $N$-dimensional linear system. Since in practis $N \ll \mathcal{N}$, the Online part is computationally very inexpensive. 


\subsection{Online multiple scattering iterative algorithm}

Given a reference shape $\widehat{D}$ and having carried out the (computationally intensive) offline procedure as described in Section 4.1, we now seek to solve the actual multiple scattering problem online for (i) any choice of parameter in $\mu \in \mathcal{D}$ (with components being the wavenumber, incident direction, and polarization of the incident wave); (ii) any configuration consisting of a number of well separated J obstacles (with each being a stretch/shrink, rotation, and translation of $\widehat{D}$ ); and hence (iii) compute the RCS of the configuration for any receiver direction. This online procedure is cheap and uses data from the offline computation.

We recall the electric far- and scattered-field representations of the multiple electromagnetic scattering problem in (2.9)-(2.10), for any $\boldsymbol{\mu} \in \mathcal{D}$, where $\boldsymbol{w}(\boldsymbol{\mu})=\sum_{\mathrm{i}=1}^{\mathrm{J}} \boldsymbol{w}_{\mathrm{i}}(\boldsymbol{\mu})$, with the surface current $\boldsymbol{w}_{\mathrm{i}} \in \boldsymbol{H}^{-1 / 2}\left(\operatorname{div}_{\partial \Omega_{\mathrm{i}}}, \partial \Omega_{i}\right)$ on the $\mathrm{i}$-th obstacle, for $i=1, \ldots, \mathrm{J}$, satisfying the J-coupled system in (3.1). Relying on the approximation scheme (3.23), we restrict the solution space to the previously assembled reduced basis space $\widehat{\mathbb{V}}_{N}$ (instead of $\widehat{\mathbb{V}}_{h}$ ) from Section 4.1 to get: for each fixed $\boldsymbol{\mu} \in \mathcal{D}$, and $\mathrm{j}=1, \ldots, \mathrm{J}$, find $\widehat{\boldsymbol{w}}_{N, \mathrm{j}}(\boldsymbol{\mu}) \in \widehat{\mathbb{V}}_{N}$ such that

$$
\sum_{\mathrm{j}=1}^{\mathrm{J}} \widehat{a}^{\mathrm{ij}}\left[\widehat{\boldsymbol{w}}_{N, \mathrm{j}}(\boldsymbol{\mu}), \widehat{\boldsymbol{v}}_{N} ; \boldsymbol{\mu}\right]=\hat{f}^{\mathrm{i}}\left[\widehat{\boldsymbol{v}}_{N} ; \boldsymbol{\mu}\right], \quad \forall \widehat{\boldsymbol{v}}_{N} \in \widehat{\mathbb{V}}_{N}, \quad \mathrm{i}=1, \ldots, \mathrm{J} .
$$

The physical surface current on $\partial \Omega_{\mathrm{i}}$ is recovered by applying the inverse Piola transformation $\boldsymbol{w}_{N, \mathrm{i}}=\widehat{\mathcal{P}}_{\mathrm{i}}^{-1} \widehat{\boldsymbol{w}}_{N, \mathrm{i}}$ for each $\mathrm{i}=1, \ldots, \mathrm{J}$. Alternatively, this can be interpreted as solving (3.9) using the restricted spaces $\mathbb{V}_{N, i}=\widehat{\mathcal{\mathcal { P }}}_{i}^{-1} \widehat{\mathbb{V}}_{N}$.

For any choice of $\boldsymbol{\mu} \in \mathcal{D}$, one first approach is to solve the resulting $\mathrm{J} N \times \mathrm{J} N$ system described by (4.4). Although this new reduced basis approach leads to substantial reduction in degrees of freedom compared to the standard boundary element method described in Section 3, this approach is not efficient when the number of $\mathrm{J}$ of obstacles is large.

Instead we propose an efficient online approach that exploits that this system can be solved in an iterative manner, inspired inspired the physical insight of the multiple scatterer configuration in which reflections from the rest of the scatterers impact the local scatterer many times. We express this as the surface current for each particle being represented by the series

$$
\widehat{\boldsymbol{w}}_{N, \mathrm{i}}(\boldsymbol{\mu})=\sum_{\ell=1}^{\infty} \widehat{\boldsymbol{w}}_{N, \mathrm{i}}^{(\ell)}(\boldsymbol{\mu}), \quad \mathrm{i}=1, \ldots, \mathrm{J},
$$

where the sequence of $\widehat{\boldsymbol{w}}_{N, \mathrm{j}}^{(\ell)}(\boldsymbol{\mu})$ satisfies

$$
\widehat{a}^{\mathrm{ii}}\left[\widehat{\boldsymbol{w}}_{N, \mathrm{i}}^{(1)}, \widehat{\boldsymbol{v}}_{N} ; \boldsymbol{\mu}\right]=\hat{f}^{\mathrm{i}}\left[\widehat{\boldsymbol{v}}_{N} ; \boldsymbol{\mu}\right], \quad \forall \widehat{\boldsymbol{v}}_{N} \in \widehat{\mathbb{V}}_{N}, \quad \mathrm{i}=1, \ldots, \mathrm{J} .
$$

and

$$
\widehat{a}^{\mathrm{i} i}\left[\widehat{\boldsymbol{w}}_{N, \mathrm{i}}^{(\ell+1)}, \widehat{\boldsymbol{v}}_{N} ; \boldsymbol{\mu}\right]=-\sum_{\substack{j=1 \\ j \neq \mathrm{i}}}^{\mathrm{J}} \widehat{a}^{\mathrm{ij}}\left[\widehat{\boldsymbol{w}}_{N, \mathrm{i}}^{(\ell)}, \widehat{\boldsymbol{v}}_{N} ; \boldsymbol{\mu}\right] \quad \forall \widehat{\boldsymbol{v}}_{N} \in \widehat{\mathbb{V}}_{N}, \quad \ell=1,2,3, \ldots
$$

We truncate this series for a finite number $L$ of reflections since in practice, for well separated obstacles, the intensity of such reflections will reduce after some iterated reflections. Notice that each reflection $\widehat{\boldsymbol{w}}_{N, \mathrm{i}}^{(\ell)}$ can be expressed in terms of the reduced basis $\left\{\xi_{n}\right\}_{n=1}^{N}$, thus

$$
\widetilde{\boldsymbol{w}}_{N, \mathrm{i}}^{(\ell)}(\boldsymbol{x} ; \boldsymbol{\mu})=\sum_{n=1}^{N} \mathrm{w}_{n, \mathrm{i}}^{(\ell)}(\boldsymbol{\mu}) \boldsymbol{\xi}_{n}(\boldsymbol{x}) .
$$

The series is truncated for a value of $L$ such that for a prescribed tolerance level tol,

$$
\max _{1 \leq i \leq j} \max _{1 \leq n \leq N}\left|\mathrm{w}_{n, \mathrm{i}}^{(L)}(\boldsymbol{\mu})-\mathrm{w}_{n, \mathrm{i}}^{(L-1)}(\boldsymbol{\mu})\right|<\text { tol }
$$

and define the iterative reduced basis approximation (on the reference surface) as

$$
\widehat{\boldsymbol{w}}_{N, L}(\boldsymbol{x} ; \boldsymbol{\mu})=\sum_{j=1}^{\mathrm{J}} \sum_{l=1}^{L} \widehat{\boldsymbol{w}}_{N, \mathrm{i}}^{(\ell)}(\boldsymbol{x} ; \boldsymbol{\mu}), \quad \boldsymbol{x} \in \partial \Omega=\bigcup_{\mathrm{j}=1}^{\mathrm{J}} \partial \Omega_{\mathrm{j}} .
$$


Having computed the solutions $\widehat{\boldsymbol{w}}_{N, \mathrm{i}}^{(\ell)}$ of (4.8), using (4.6)-(4.7), we compute the reduced basis approximation to the surface current in (2.9)-(2.10), as

$$
\boldsymbol{w}_{N, L}(\boldsymbol{x} ; \boldsymbol{\mu})=\sum_{j=1}^{\mathrm{J}} \sum_{l=1}^{L}\left(\widehat{\mathcal{P}}_{\mathrm{i}}^{-1} \widehat{\boldsymbol{w}}_{N, \mathrm{i}}^{(\ell)}\right)(\boldsymbol{x} ; \boldsymbol{\mu}), \quad \boldsymbol{x} \in \partial \Omega=\bigcup_{\mathrm{j}=1}^{\mathrm{J}} \partial \Omega_{\mathrm{j}} .
$$

The final task in the online process is the computation of the RCS of the configuration for any parameter $\widetilde{\boldsymbol{d}}_{\mathrm{rcs}}$, using (5.46). We compute the approximation to the RCS of the total configuration as

$$
\operatorname{RCS}\left[\boldsymbol{w}_{N, L} ; \widetilde{\boldsymbol{d}}_{\mathrm{rcs}}, \boldsymbol{\mu}\right]=10 \log _{10}\left(4 \pi \frac{\left|\boldsymbol{E}_{\infty}\left[\boldsymbol{w}_{N, L} ; \widetilde{\boldsymbol{d}}_{\mathrm{rcs}}, \boldsymbol{\mu}\right]\right|^{2}}{\left|\boldsymbol{p}_{\text {inc }}\right|^{2}}\right),
$$

based on (2.9).

\section{Decompositions of parameter dependent operators}

As for the single obstacle case, described in section 4.1, a crucial ingredient in the efficient solution of the iterative reduced basis scheme (4.6)-(4.7), i.e., independent on $\mathcal{N}=\operatorname{dim}\left(\widehat{\mathbb{V}}_{h}\right)$, is the affine decomposition to separate the parameter and $\boldsymbol{x}$-variable dependence (analogous to (4.2)-(4.3)) in the sesquilinear and linear forms involved. Note that without an affine decomposition, the forms involved in (4.6)-(4.7) need to be reassembled for each new parameter value. This process depends on $\mathcal{N}$. The affine decomposition allows to precompute certain parameter independent quantities (that are expensive to compute since the operation count depends on $\mathcal{N}$ ) in the Offline stage once and for all.

As mentioned previously, such an affine decomposition is not feasible in our case and we need to seek an approximation to enable this. For this, the Empirical Interpolation Method (EIM) [28, 29] plays a crucial role as it allows is express the operators and sources through an affine decomposition to any accuracy needed for the application of interest. In the general setting, let $\boldsymbol{\mu}$ be a generic parameter value contained in a chosen parameter set. The EIM is a tool to approximate parameter dependent functions $\mathcal{F}(\cdot ; \boldsymbol{\mu})$, to any required accuracy, using an affine decomposition of the form

$$
\mathcal{F}(\cdot ; \boldsymbol{\mu}) \approx(\mathcal{I} \mathcal{F})(\cdot ; \boldsymbol{\mu})=\sum_{p=1}^{M} \beta_{p}(\boldsymbol{\mu}) \mathcal{F}\left(\cdot ; \boldsymbol{\mu}_{p}\right)
$$

where the parameter sample values $\left\{\boldsymbol{\mu}_{p}\right\}$ in the EIM are chosen by a greedy algorithm and for each new parameter value $\boldsymbol{\mu}$, the coefficients $\beta_{p}(\boldsymbol{\mu})$ are obtained by solving a lower triangular $M$ dimensional linear system, see [28, 29] for details.

In the following, we explain in detail how each form involved in (4.6)-(4.7) and (5.47) can be approximated using this approach.

\subsection{Decomposition of sesquilinear forms}

For any fixed $\boldsymbol{\mu} \in \mathcal{D}$, and $i, j=1, \ldots, \mathrm{J}$ with $i \neq j$, interaction between the $\mathrm{J}$ obstacles in the configuration, through electromagnetic waves, are represented by the $\mathrm{J}^{2}-\mathrm{J}$ sesquilinear forms $\widehat{\boldsymbol{a}}^{\mathrm{ij}}[\widehat{\boldsymbol{\psi}}, \widehat{\boldsymbol{\eta}} ; \boldsymbol{\mu}]: \boldsymbol{H}^{-1 / 2}\left(\operatorname{div}_{\partial \widehat{D}}, \partial \widehat{D}\right) \times$ $\boldsymbol{H}^{-1 / 2}\left(\operatorname{div}_{\partial \widehat{D}}, \partial \widehat{D}\right) \rightarrow \mathbb{C}$, defined in (3.20). The parameters governing these forms are: (i) wavenumber $(k)$; (ii) stretch/shrink $\left(\gamma_{i}, \gamma_{j}\right)$; (iii) rotation $\left(\mathrm{B}_{i}, \mathrm{~B}_{\mathrm{j}}\right)$; and (iv) translation $\left(\boldsymbol{b}_{i}, \boldsymbol{b}_{j}\right)$.

For $\widehat{\boldsymbol{\psi}}, \widehat{\boldsymbol{\eta}} \in \boldsymbol{H}^{-1 / 2}\left(\operatorname{div}_{\partial \widehat{D}}, \partial \widehat{D}\right)$, one easily sees that

$$
\left.\left(\mathrm{B}_{\mathrm{j}} \widehat{\boldsymbol{w}}(\hat{\boldsymbol{y}})\right) \cdot\left(\mathrm{B}_{\mathrm{i}} \overline{\widehat{\boldsymbol{v}}(\hat{\boldsymbol{x}})}\right)=\sum_{l, n=1}^{3}[\widehat{\boldsymbol{w}}(\hat{\boldsymbol{y}})]_{l}\left[\mathrm{~B}_{\mathrm{ij}}\right]_{l, n} \overline{\widehat{\hat{\boldsymbol{v}}(\hat{\boldsymbol{x}})}}\right]_{n},
$$

where $\mathrm{B}_{i j}:=\mathrm{B}_{i}^{T} \mathrm{~B}_{j} \in S O(3)$ is also a rotation matrix. Hence the key to get the affine decomposition of the interactive sesquilinear forms (3.20) is to get an additive variable and parameter separation of the non-singular kernel functions

$$
\widehat{\Phi}_{k}^{\mathrm{ij}}(\hat{\boldsymbol{x}}, \hat{\boldsymbol{y}})=\Phi_{k}\left(T_{\mathrm{i}}(\hat{\boldsymbol{x}}), T_{\mathrm{j}}(\hat{\boldsymbol{y}})\right)=\frac{e^{i k\left|T_{\mathrm{i}}(\hat{\boldsymbol{x}})-T_{\mathrm{j}}(\hat{\boldsymbol{y}})\right|}}{4 \pi\left|T_{\mathrm{i}}(\hat{\boldsymbol{x}})-T_{\mathrm{j}}(\hat{\boldsymbol{y}})\right|}, \quad \mathrm{i}, \mathrm{j}=1, \ldots, J, \quad \mathrm{i} \neq \mathrm{j} .
$$


We have

$$
T_{\mathrm{i}}(\hat{\boldsymbol{x}})-T_{\mathrm{j}}(\hat{\boldsymbol{y}})=\gamma_{\mathrm{i}} \mathrm{B}_{\mathrm{i}} \hat{\boldsymbol{x}}+\boldsymbol{b}_{\mathrm{i}}-\gamma_{\mathrm{j}} \mathrm{B}_{\mathrm{j}} \hat{\boldsymbol{y}}-\boldsymbol{b}_{\mathrm{j}}=\gamma_{\mathrm{i}} \mathrm{B}_{\mathrm{i}}\left[\hat{\boldsymbol{x}}-\frac{\gamma_{\mathrm{j}}}{\gamma_{\mathrm{i}}} \mathrm{B}_{\mathrm{ij}} \hat{\boldsymbol{y}}+\frac{1}{\gamma_{\mathrm{i}}} \mathrm{B}_{\mathrm{i}}^{T}\left(\boldsymbol{b}_{\mathrm{i}}-\boldsymbol{b}_{\mathrm{j}}\right)\right]
$$

and hence

$$
\left|T_{i}(\hat{\boldsymbol{x}})-T_{\mathrm{j}}(\hat{\boldsymbol{y}})\right|=\gamma_{\mathrm{i}}\left|\hat{\boldsymbol{x}}-\gamma_{\mathrm{ij}} \mathrm{B}_{\mathrm{ij}} \hat{\boldsymbol{y}}+\boldsymbol{c}_{\mathrm{ij}}\right|, \quad \gamma_{\mathrm{ij}}:=\frac{\gamma_{j}}{\gamma_{\mathrm{i}}}, \quad \boldsymbol{c}_{\mathrm{ij}}:=\frac{1}{\gamma_{\mathrm{i}}} \mathrm{B}_{\mathrm{i}}^{T}\left(\boldsymbol{b}_{\mathrm{i}}-\boldsymbol{b}_{\mathrm{j}}\right)
$$

Let

$$
k \in\left[k^{-}, k^{+}\right], \quad \gamma_{\mathrm{i}} \in\left[\gamma^{-}, \gamma^{+}\right], \quad \boldsymbol{b}_{\mathrm{i}} \in B\left(R_{+}\right), \quad \mathrm{i}=1, \ldots, J,
$$

for some positive constants $k^{-}, k^{+}, \gamma^{-}, \gamma^{+}, R_{+}$, and $B\left(R_{+}\right)$is a ball of radius $R_{+}$containing the full multiple scatterer configuration $\Omega=\bigcup_{j=1}^{\mathrm{j}} \Omega_{\mathrm{j}}$. Let $0<\widehat{R_{+}}<R_{+}$be such that $B\left(\widehat{R_{+}}\right)$is the smallest ball of radius $\widehat{R_{+}}$containing the reference obstacle $\widehat{D}$.

Using (5.2)-(5.4), and for $\Omega$ consisting only of well separated obstacles, without loss of generality, we assume that

$$
\gamma^{-} k^{-} \leq \gamma_{\mathrm{i}} k \leq \gamma^{+} k^{+}, \quad \frac{\gamma^{-}}{\gamma^{+}} \leq \gamma_{\mathrm{ij}} \leq \frac{\gamma^{+}}{\gamma^{-}}, \quad c_{\mathrm{ij}} \in B\left(\frac{2 R_{+}}{\gamma^{-}}\right) \backslash B\left(\left(1+\frac{1}{\gamma^{+}}\right) \widehat{R}_{+}\right) .
$$

Consequently, we define the parametrized function

$$
\mathcal{G}(\hat{\boldsymbol{x}}, \hat{\boldsymbol{y}} ; k, \gamma, \mathrm{B}, \boldsymbol{c})=\frac{e^{i k|\hat{\boldsymbol{x}}-\gamma \mathrm{B} \hat{\boldsymbol{y}}+\boldsymbol{c}|}}{4 \pi|\hat{\boldsymbol{x}}-\gamma \mathrm{B} \hat{\boldsymbol{y}}+\boldsymbol{c}|}, \quad \hat{\boldsymbol{x}}, \hat{\boldsymbol{y}} \in \widehat{D}, \quad(k, \gamma, \mathrm{B}, \boldsymbol{c}) \in \mathcal{D}_{1},
$$

where the form parameter space $\mathcal{D}_{1} \subset \mathbb{R}^{7}$ is given by

$$
\mathcal{D}_{1}=\left[k^{-} \gamma^{-}, k^{+} \gamma^{+}\right] \times\left[\frac{\gamma^{-}}{\gamma_{+}}, \frac{\gamma_{+}}{\gamma_{-}}\right] \times S O(3) \times\left[B\left(\frac{2 R_{+}}{\gamma^{-}}\right) \backslash B\left(\left(1+\frac{1}{\gamma^{+}}\right) \widehat{R}_{+}\right)\right] .
$$

Using (5.2), (5.3), and (5.6),

$$
\widehat{\Phi}_{k}^{\mathrm{ij}}(\hat{\boldsymbol{x}}, \hat{\boldsymbol{y}})=\frac{e^{i k\left|T_{\mathrm{i}}(\hat{\boldsymbol{x}})-T_{\mathrm{j}}(\hat{\boldsymbol{y}})\right|}}{4 \pi\left|T_{\mathrm{i}}(\hat{\boldsymbol{x}})-T_{\mathrm{j}}(\hat{\boldsymbol{y}})\right|}=\gamma_{\mathrm{i}}^{-1} \mathcal{G}\left(\hat{\boldsymbol{x}}, \hat{\boldsymbol{y}} ; k \gamma_{\mathrm{i}}, \gamma_{\mathrm{ij}}, \mathrm{B}_{\mathrm{ij}}, \boldsymbol{c}_{\mathrm{ij}}\right) .
$$

Applying the EIM to the non-singular kernel $\mathcal{G}(\cdot, \cdot ; k, \gamma, \mathrm{B}, \boldsymbol{c})$ yields $M_{\mathcal{G}}$ sample points $\left\{k^{m}, \gamma^{m}, \mathrm{~B}^{m}, \boldsymbol{c}^{m}\right\}_{m=1}^{M_{\mathcal{G}}} \in \mathcal{D}_{1}$ and $M_{\mathcal{G}}$ coefficient functions defined on $\mathcal{D}_{1}$ and hence the interpolant

$$
(\mathcal{I} \mathcal{G})(\hat{\boldsymbol{x}}, \hat{\boldsymbol{y}} ; k, \gamma, \mathrm{B}, \boldsymbol{c})=\sum_{m=1}^{M_{\mathcal{G}}} \alpha_{m}^{\mathcal{G}}(k, \gamma, \mathrm{B}, \boldsymbol{c}) \mathcal{G}_{m}(\boldsymbol{x}, \boldsymbol{y}), \quad \mathcal{G}_{m}(\boldsymbol{x}, \boldsymbol{y}):=\mathcal{G}\left(\boldsymbol{x}, \boldsymbol{y} ; k^{m}, \gamma^{m}, \mathrm{~B}^{m}, \boldsymbol{c}^{m}\right)
$$

Using the EIM interpolant, we approximate the interactive kernel (5.8), to any required accuracy, as

$$
\widehat{\Phi}_{k}^{\mathrm{ij}}(\hat{\boldsymbol{x}}, \hat{\boldsymbol{y}}) \approx \widehat{\Phi}_{k, M_{\mathcal{G}}}^{\mathrm{ij}}(\hat{\boldsymbol{x}}, \hat{\boldsymbol{y}}):=\gamma_{\mathrm{i}}^{-1}(\mathcal{I} \mathcal{G})(\hat{\boldsymbol{x}}, \hat{\boldsymbol{y}} ; k, \gamma, \mathrm{B}, \boldsymbol{c}), \quad \hat{\boldsymbol{x}}, \hat{\boldsymbol{y}} \in \widehat{D}, \quad(k, \gamma, \mathrm{B}, \boldsymbol{c}) \in \mathcal{D}_{1} .
$$

For $i, j=1, \ldots, \mathrm{J}$, approximating $\widehat{\Phi}_{k}^{\mathrm{ij}}$ in (3.20) by $\widehat{\Phi}_{k, M_{\mathcal{G}}}^{\mathrm{ij}}$ and using (5.1), we get

$$
\widehat{a}^{\mathrm{ij}}[\widehat{\psi}, \widehat{\boldsymbol{\eta}} ; \boldsymbol{\mu}] \approx \widehat{a}_{M_{\mathcal{G}}}^{\mathrm{ij}}[\widehat{\psi}, \widehat{\boldsymbol{\eta}} ; \boldsymbol{\mu}], \quad \boldsymbol{\mu} \in \mathcal{D}_{1}
$$

where for $\widehat{\boldsymbol{\psi}}, \widehat{\boldsymbol{\eta}} \in \boldsymbol{H}^{-1 / 2}\left(\operatorname{div}_{\partial \widehat{D}}, \partial \widehat{D}\right), i, j=1, \ldots, \mathrm{J}$, and $\mathrm{i} \neq \mathrm{j}$,

$$
\begin{aligned}
& \widehat{a}_{M_{\mathcal{G}}}^{\mathrm{ij}}[\widehat{\boldsymbol{\psi}}, \widehat{\boldsymbol{\eta}} ; \boldsymbol{\mu}] \\
& =i k Z \gamma_{\mathrm{j}} \sum_{m=1}^{M_{\mathcal{G}}} \alpha_{m}^{\mathcal{G}}\left(k \gamma_{\mathrm{i}}, \gamma_{\mathrm{ij}}, \mathrm{B}_{\mathrm{ij}}, \boldsymbol{c}_{\mathrm{ij}}\right)\left\{\sum_{t, \bar{t}=1}^{3}\left(\mathrm{~B}_{\mathrm{j}}^{T} \mathrm{~B}_{\mathrm{i}}\right)_{t, \bar{t}}[\widehat{R 1}[\widehat{\boldsymbol{\psi}}, \widehat{\boldsymbol{\eta}} ; m]]_{t, \bar{t}}-\frac{1}{k^{2} \gamma_{\mathrm{i}} \gamma_{\mathrm{j}}} \widehat{R 2}[\widehat{\boldsymbol{\psi}}, \widehat{\boldsymbol{\eta}} ; m]\right\},
\end{aligned}
$$


and for $m=1, \ldots M_{\mathcal{G}}, l, n=1,2,3$

$$
\begin{aligned}
{[\widehat{R 1}[\widehat{\boldsymbol{\psi}}, \widehat{\boldsymbol{\eta}} ; m]]_{l, n} } & =\int_{\partial \widehat{D}} \int_{\partial \widehat{D}} \mathcal{G}_{m}(\boldsymbol{x}, \boldsymbol{y})[\widehat{\boldsymbol{\psi}}(\hat{\boldsymbol{y}})]_{l}[\overline{\widehat{\boldsymbol{\eta}}(\hat{\boldsymbol{x}})}]_{n} d s(\hat{\boldsymbol{y}}) d s(\hat{\boldsymbol{x}}), \\
\widehat{R 2}[\widehat{\boldsymbol{\psi}}, \widehat{\boldsymbol{\eta}} ; m] & =\int_{\partial \widehat{D}} \int_{\partial \widehat{D}} \mathcal{G}_{m}(\boldsymbol{x}, \boldsymbol{y}) \operatorname{div}_{\hat{\boldsymbol{y}}} \widehat{\boldsymbol{w}}(\hat{\boldsymbol{y}}) \operatorname{div}_{\hat{\boldsymbol{x}}} \overline{\widehat{\boldsymbol{v}}(\hat{\boldsymbol{x}})} d s(\hat{\boldsymbol{y}}) d s(\hat{\boldsymbol{x}}) .
\end{aligned}
$$

It is important to note that the sesquilinear forms in (5.13)-(5.14) are independent of the number, shape, location of the obstacles in the multiple scatterer configuration and the wavenumber $k$. If we replace $\psi, \boldsymbol{\eta}$ in (5.13)-(5.14) by finitely many basis functions, the associated matrices can be computed in parallel (with respect to the $M_{\mathcal{G}}$ EIM sample points) before simulating the multiple electromagnetic wave interactions for any parameter $\boldsymbol{\mu} \in \mathcal{D}$ and number of scatterers in the configuration.

Remark 4. In the simplified case where the geometry of the multiple scatterer depends only of translations (that is, $\mathrm{B}_{\mathrm{i}}=I, \gamma_{\mathrm{i}}=1$, for all $\left.\mathrm{i}=1, \ldots, J\right)$, for the above EIM we can define a simpler parameter dependent function

$$
\mathcal{G}(\boldsymbol{x} ; k, \boldsymbol{c})=\frac{e^{i k|x+c|}}{4 \pi|\boldsymbol{x}+\boldsymbol{c}|}
$$

with $\boldsymbol{x} \in B\left(2 \widehat{R}_{+}\right)$and $\boldsymbol{c} \in B\left(R_{+}\right) \backslash B\left(2 \widehat{R}_{+}\right)$. The affine decomposition is then satisfied in a similar but simpler form.

\subsection{Decomposition of the forms of the individual scatterers}

We consider the mass sesquilinear forms $\widehat{a}^{i \mathrm{i}}(\widehat{\boldsymbol{w}}, \widehat{\boldsymbol{v}} ; \boldsymbol{\mu})$ for $\mathrm{i}=1, \ldots, \mathrm{J}$. This is a simpler case as compared to the one discussed in the previous section. For $i=1, \ldots, \mathrm{J}$, we have

$$
\widehat{\Phi}_{k}^{\mathrm{ii}}(\hat{\boldsymbol{x}}, \hat{\boldsymbol{y}})=\Phi_{k}\left(T_{\mathrm{i}}(\hat{\boldsymbol{x}}), T_{\mathrm{i}}(\hat{\boldsymbol{y}})\right)=\frac{e^{i k\left|T_{\mathrm{i}}(\hat{\boldsymbol{x}})-T_{\mathrm{i}}(\hat{\boldsymbol{y}})\right|}}{4 \pi\left|T_{\mathrm{i}}(\hat{\boldsymbol{x}})-T_{\mathrm{i}}(\hat{\boldsymbol{y}})\right|}=\frac{e^{i k \gamma_{\mathrm{i}}|\hat{x}-\hat{\boldsymbol{y}}|}}{4 \pi \gamma_{\mathrm{i}}|\hat{\boldsymbol{x}}-\hat{\boldsymbol{y}}|}=\gamma_{\mathrm{i}}^{-1} \mathcal{M}\left(|\hat{\boldsymbol{x}}-\hat{\boldsymbol{y}}| ; \gamma_{\mathrm{i}} k\right),
$$

where

We have

$$
\mathcal{M}\left(r ; k^{\star}\right)=\frac{e^{i k^{\star} r}}{4 \pi r}, \quad r \in\left[0,2 \widehat{R}_{+}\right], \quad k^{\star} \in\left[\gamma^{-} k^{-}, \gamma^{+} k^{+}\right]
$$

$$
\mathcal{M}\left(r ; k^{\star}\right)=\mathcal{M}(r ; 0)+\mathcal{M}_{1}\left(r ; k^{\star}\right), \quad \mathcal{M}_{1}\left(r ; k^{\star}\right):=\frac{e^{i k^{\star} r}-1}{4 \pi r},
$$

with only the non-singular part $\mathcal{M}_{1}\left(r ; k^{\star}\right)$ of the kernel $\mathcal{M}\left(r ; k^{\star}\right)$ depending on the parameter. Applying the EIM to $\mathcal{M}_{1}\left(\cdot ; k^{\star}\right)$ yields $M_{\mathcal{M}}$ sample points $k_{m} \in\left[\gamma^{-} k^{-}, \gamma^{+} k^{+}\right]$and coefficient functions $\alpha_{m}^{\mathcal{M}}\left(k^{\star}\right)$, for $m=1, \ldots, M_{\mathcal{M}}$. Thus with $k_{0}=0, \alpha_{0}\left(k^{\star}\right)=1$, we obtain the EIM-based approximation of the singular kernel (of the reference obstacle)

$$
\mathcal{M}\left(r ; k^{\star}\right) \approx(\mathcal{I} \mathcal{M})\left(r ; k^{\star}\right)=\sum_{m=0}^{M_{\mathcal{M}}} \alpha_{m}^{\mathcal{M}}\left(k^{\star}\right) \mathcal{M}_{m}(r), \quad \mathcal{M}_{m}(r):=\mathcal{M}\left(r ; k_{m}\right) .
$$

Hence, for $i=1, \ldots, \mathrm{J}$, we approximate the sesquilinear form corresponding to the $i$-th scatterer in the configuration as

$$
a^{\mathrm{i} \mathrm{i}}[\widehat{\boldsymbol{\psi}}, \widehat{\boldsymbol{\eta}} ; \boldsymbol{\mu}] \approx \widehat{a}_{M_{\mathcal{M}}}^{\mathrm{ii}}[\widehat{\boldsymbol{\psi}}, \widehat{\boldsymbol{\eta}} ; \boldsymbol{\mu}]=i k Z \gamma_{\mathrm{i}} \sum_{m=0}^{M_{\mathcal{M}}} \alpha_{m}^{\mathcal{M}}\left(k \gamma_{\mathrm{i}}\right)\left\{\widehat{a} 1[\widehat{\boldsymbol{\psi}}, \widehat{\boldsymbol{\eta}} ; m]-\frac{1}{k^{2} \gamma_{\mathrm{i}}^{2}} \widehat{a}[-\widehat{\boldsymbol{\psi}}, \widehat{\boldsymbol{\eta}} ; m]\right\},
$$

where for $m=0, \ldots, M_{\mathcal{M}}$,

$$
\begin{aligned}
& \widehat{a} 1 \widehat{\boldsymbol{\psi}}, \widehat{\boldsymbol{\eta}} ; m]=\int_{\partial \widehat{D}} \int_{\partial \widehat{D}} \mathcal{M}_{m}\left(|\boldsymbol{x}-\boldsymbol{y}| ; k_{m}\right) \widehat{\boldsymbol{\psi}}(\hat{\boldsymbol{y}}) \overline{\widehat{\boldsymbol{\eta}}(\hat{\boldsymbol{x}})} d s(\hat{\boldsymbol{y}}) d s(\hat{\boldsymbol{x}}), \\
& \widehat{a}[\widehat{\boldsymbol{\psi}}, \widehat{\boldsymbol{\eta}} ; m]=\int_{\partial \widehat{D}} \int_{\partial \widehat{D}} \mathcal{M}_{m}\left(|\boldsymbol{x}-\boldsymbol{y}| ; k_{m}\right) \operatorname{div}_{\hat{\boldsymbol{y}}} \widehat{\boldsymbol{w}}(\hat{\boldsymbol{y}}) \operatorname{div}_{\hat{\boldsymbol{x}}} \overline{\widehat{\boldsymbol{v}}(\hat{\boldsymbol{x}})} d s(\hat{\boldsymbol{y}}) d s(\hat{\boldsymbol{x}}) .
\end{aligned}
$$


If we consider the single obstacle electromagnetic scattering problem with $\widehat{D}$ being the scatterer (and the wavenumber parameter domain in $\left[\gamma^{-} k^{-}, \gamma^{+} k^{+}\right]$) and apply the EIM to the associated electric field integral equation sesquilinear form in two parts, the resulting sesquilinear forms (that are independent of the wavenumber) are precisely those in (5.20)-(5.21). Thus, if $\widehat{a}[\widehat{\boldsymbol{\psi}}, \widehat{\boldsymbol{\eta}} ; \boldsymbol{\mu}]$ is the sesquilinear form for electromagnetic scattering by the single obstacle $\widehat{D}$, then for $\widehat{\boldsymbol{\psi}}, \widehat{\boldsymbol{\eta}} \in \boldsymbol{H}^{-1 / 2}\left(\operatorname{div}_{\partial \widehat{D}}, \partial \widehat{D}\right)$,

$$
\left.\widehat{a}[\widehat{\boldsymbol{\psi}}, \widehat{\boldsymbol{\eta}} ; \boldsymbol{\mu}]=i k Z \int_{\partial \widehat{D}} \int_{\partial \widehat{D}} \Phi_{k}(\hat{\boldsymbol{x}}, \hat{\boldsymbol{y}})(\widehat{(\boldsymbol{\psi}}(\hat{\boldsymbol{y}})) \cdot(\overline{\boldsymbol{\boldsymbol { \eta }}(\hat{\boldsymbol{x}})})-\frac{1}{k^{2}} \operatorname{div}_{\hat{\boldsymbol{y}}} \widehat{\boldsymbol{\psi}}(\hat{\boldsymbol{y}}) \operatorname{div}_{\hat{\boldsymbol{x}}} \overline{\widehat{\boldsymbol{\eta}}(\hat{\boldsymbol{x}})}\right) d s(\hat{\boldsymbol{y}}) d s(\hat{\boldsymbol{x}}),
$$

and the associated affine decomposition is given by

$$
\widehat{a}[\widehat{\boldsymbol{\psi}}, \widehat{\boldsymbol{\eta}} ; \boldsymbol{\mu}] \approx \widehat{a}_{M_{\mathcal{M}}}[\widehat{\boldsymbol{\psi}}, \widehat{\boldsymbol{\eta}} ; \boldsymbol{\mu}]=i k Z \sum_{m=0}^{M_{\mathcal{M}}} \alpha_{m}^{\mathcal{M}}(k)\left\{\widehat{a 1}[\widehat{\boldsymbol{\psi}}, \widehat{\boldsymbol{\eta}} ; m]-\frac{1}{k^{2}} \widehat{a} 2[\widehat{\boldsymbol{\psi}}, \widehat{\boldsymbol{\eta}} ; m]\right\}
$$

\subsection{Decomposition of the input source term}

Motivated by the representation of the incident field in (2.1), we consider the scalar valued function

$$
\mathcal{F}\left(\hat{\boldsymbol{x}} ; k, \widetilde{\boldsymbol{d}}_{\mathrm{inc}}\right)=e^{i k \hat{\boldsymbol{x}} \cdot \widetilde{\boldsymbol{d}}_{\mathrm{inc}}}, \quad \hat{\boldsymbol{x}} \in \widehat{D}, \quad\left(k, \widetilde{\boldsymbol{d}}_{\mathrm{inc}}\right) \in\left[\gamma^{-} k^{-}, \gamma^{+} k^{+}\right] \times S^{2},
$$

and its EIM approximation

$$
\mathcal{F}\left(\hat{\boldsymbol{x}} ; k, \widetilde{\boldsymbol{d}}_{\mathrm{inc}}\right) \approx(\mathcal{I} \mathcal{F})\left(\hat{\boldsymbol{x}} ; k, \widetilde{\boldsymbol{d}}_{\mathrm{inc}}\right)=\sum_{m=1}^{M_{\mathcal{F}}} \alpha_{m}^{\mathcal{F}}\left(k, \widetilde{\boldsymbol{d}}_{\mathrm{inc}}\right) e^{i k_{m} \hat{\boldsymbol{x}} \cdot \widetilde{\boldsymbol{d}}_{m}}
$$

For $\mathrm{i}=1, \ldots, \mathrm{J}$, using $T_{\mathrm{i}}(\hat{\boldsymbol{x}})=\gamma_{\mathrm{i}} \mathrm{B}_{\mathrm{i}} \hat{\boldsymbol{x}}+\boldsymbol{b}_{\mathrm{i}}$, the incident wave part of the integrand in (3.22) has the form

$$
\mathrm{B}_{\mathrm{i}}^{T} \boldsymbol{E}^{\mathrm{inc}}\left(T_{\mathrm{i}}(\hat{\boldsymbol{x}}) ; \boldsymbol{\mu}\right)=\mathrm{B}_{\mathrm{i}}^{T} \boldsymbol{p}_{\mathrm{inc}} e^{i k \boldsymbol{b}_{\mathrm{i}} \cdot \widetilde{\boldsymbol{d}}_{\mathrm{inc}}} e^{i k \gamma_{\mathrm{i}} \mathrm{B}_{\mathrm{i}} \hat{\boldsymbol{x}} \cdot \widetilde{\boldsymbol{d}}_{\mathrm{inc}}}=\mathrm{B}_{\mathrm{i}}^{T} \boldsymbol{p}_{\mathrm{inc}} e^{i k \boldsymbol{b}_{\mathrm{i}} \cdot \widetilde{\boldsymbol{d}}_{\mathrm{inc}}} e^{i k \gamma_{\mathrm{i}} \hat{\boldsymbol{x}} \cdot \mathrm{B}_{\mathrm{i}}^{T} \widetilde{\boldsymbol{d}}_{\mathrm{inc}}} .
$$

Hence using (5.25) in (5.26), we obtain

$$
\mathrm{B}_{\mathrm{i}}^{T} \boldsymbol{E}^{\mathrm{inc}}\left(T_{\mathrm{i}}(\hat{\boldsymbol{x}}) ; \boldsymbol{\mu}\right) \approx \mathrm{B}_{\mathrm{i}}^{T} \boldsymbol{p}_{\mathrm{inc}} e^{i k \boldsymbol{b}_{\mathrm{i}} \cdot \widetilde{\boldsymbol{d}}_{\mathrm{inc}}} \sum_{m=1}^{M_{\mathcal{F}}} \alpha_{m}^{\mathcal{F}}\left(\gamma_{\mathrm{i}} k, \mathrm{~B}_{\mathrm{i}}^{T} \widetilde{\boldsymbol{d}}_{\mathrm{inc}}\right) e^{i k_{m} \hat{\boldsymbol{x}} \cdot \widetilde{\boldsymbol{d}}_{m}} .
$$

The source term in (3.22) can be approximated using the affine decomposition

$$
\widehat{f^{\mathrm{i}}}[\widehat{\boldsymbol{\eta}} ; \boldsymbol{\mu}] \approx \widehat{f}_{M_{\mathcal{F}}^{\mathrm{i}}}^{\mathrm{\eta}}[\widehat{\boldsymbol{\eta}} ; \boldsymbol{\mu}]=-\sum_{m=1}^{M_{\mathcal{F}}} \gamma_{\mathrm{i}} e^{i k \boldsymbol{b}_{\mathrm{i}} \cdot \widetilde{\boldsymbol{d}}_{\mathrm{inc}}} \alpha_{m}^{\mathcal{F}}\left(\gamma_{\mathrm{i}} k, \mathrm{~B}_{\mathrm{i}}^{T} \widetilde{\boldsymbol{d}}_{\mathrm{inc}}\right) \mathrm{B}_{\mathrm{i}}^{T} \boldsymbol{p}_{\mathrm{inc}} \cdot \widehat{\mathbf{f}}[\widehat{\boldsymbol{\eta}} ; m],
$$

where

$$
\widehat{\mathbf{f}} \widehat{\boldsymbol{\eta}} ; m]=\int_{\partial \widehat{D}} e^{i k_{m} \hat{\boldsymbol{x}} \cdot \widetilde{\boldsymbol{d}}_{m}} \overline{\widehat{\boldsymbol{\eta}}(\hat{\boldsymbol{x}})} d s(\hat{\boldsymbol{x}}), \quad m=1, \ldots, M_{\mathcal{F}}
$$

As in Section 5.2, it is important to observe that for electromagnetic scattering by the single obstacle $\widehat{D}$, with source function denoted by $\widehat{f}[\widehat{\boldsymbol{\eta}} ; \boldsymbol{\mu}]$, for $\widehat{\boldsymbol{\eta}} \in \boldsymbol{H}^{-1 / 2}\left(\operatorname{div}_{\partial \widehat{D}}, \partial \widehat{D}\right)$, we have

$$
\widehat{f}[\widehat{\boldsymbol{\eta}} ; \boldsymbol{\mu}]=-\int_{\partial \widehat{D}} \boldsymbol{E}^{\mathrm{inc}}(\hat{\boldsymbol{x}} ; \boldsymbol{\mu}) \cdot \overline{\widehat{\boldsymbol{\eta}}(\hat{\boldsymbol{x}})} d s(\hat{\boldsymbol{x}}),
$$

and its associated EIM-based affine decomposition is given by

$$
\widehat{f}[\widehat{\boldsymbol{\eta}} ; \boldsymbol{\mu}] \approx \widehat{f}_{M_{\mathcal{F}}}[\widehat{\boldsymbol{\eta}} ; \boldsymbol{\mu}]=-\sum_{m=1}^{M_{\mathcal{F}}} \alpha_{m}^{\mathcal{F}}\left(k, \widetilde{\boldsymbol{d}}_{\text {inc }}\right) \boldsymbol{p}_{\text {inc }} \cdot \widehat{\mathbf{f}}[\widehat{\boldsymbol{\eta}} ; m]
$$




\subsection{Decomposition of output RCS functionals}

The main parameter dependent output functional of interest in electromagnetic scattering is the radar signature. Recall from (2.8)-(2.9) that the surface current dependent RCS is given by

$$
\operatorname{RCS}\left[\boldsymbol{w} ; \widetilde{\boldsymbol{d}}_{\mathrm{rcs}}, \boldsymbol{\mu}\right]=10 \log _{10}\left(\frac{4 \pi\left|\boldsymbol{E}_{\infty}\left[\boldsymbol{w} ; \widetilde{\boldsymbol{d}}_{\mathrm{rcs}}, \boldsymbol{\mu}\right]\right|^{2}}{\left|\boldsymbol{p}_{\mathrm{inc}}\right|^{2}}\right)
$$

where $\boldsymbol{E}_{\infty}$ is a linear functional of the unique surface current solution of (2.13), given by

$$
\boldsymbol{E}_{\infty}\left[\boldsymbol{w} ; \widetilde{\boldsymbol{d}}_{\mathrm{rcs}}, \boldsymbol{\mu}\right]=\sum_{j=1}^{J} i k Z \int_{\partial \Omega_{\mathrm{j}}} \widetilde{\boldsymbol{d}}_{\mathrm{rcs}} \times\left(\boldsymbol{w}_{\mathrm{j}}(\boldsymbol{x}) \times \widetilde{\boldsymbol{d}}_{\mathrm{rcs}}\right) e^{i k \boldsymbol{x} \cdot \widetilde{\boldsymbol{d}}_{\mathrm{rcs}}} d s(\boldsymbol{x})=: \sum_{j=1}^{J} \boldsymbol{E}_{\infty}^{j}\left[\boldsymbol{w}_{\mathrm{j}} ; \widetilde{\boldsymbol{d}}_{\mathrm{rcs}}, \boldsymbol{\mu}\right] .
$$

Using the transformation $T_{j}$ and writing $\boldsymbol{x} \in \partial \Omega_{j}$ as $\boldsymbol{x}=\gamma_{\mathrm{j}} \mathrm{B}_{\mathrm{j}} \hat{\boldsymbol{x}}+\boldsymbol{b}_{\mathrm{j}}$, and $\boldsymbol{w}_{\mathrm{j}} \in \boldsymbol{H}^{-1 / 2}\left(\operatorname{div}_{\partial \Omega_{\mathrm{j}}}, \partial \Omega_{\mathrm{j}}\right)$ as $\boldsymbol{w}_{\mathrm{j}}=\widehat{\mathcal{P}}_{\mathrm{j}}^{-1} \widehat{\boldsymbol{w}}$, we get

$$
\begin{aligned}
& \boldsymbol{E}_{\infty}^{\mathrm{j}}\left[\boldsymbol{w}_{\mathrm{j}} ; \widetilde{\boldsymbol{d}}_{\mathrm{rcs}}, \boldsymbol{\mu}\right] \\
& =\widehat{\boldsymbol{E}}_{\infty}^{\mathrm{j}}\left[\widehat{\boldsymbol{w}} ; \widetilde{\boldsymbol{d}}_{\mathrm{rcs}}, \boldsymbol{\mu}\right]=i k Z \gamma_{\mathrm{j}} e^{i k \boldsymbol{b}_{\mathrm{j}} \cdot \widetilde{\boldsymbol{d}}_{\mathrm{rcs}}} \widetilde{\boldsymbol{d}}_{\mathrm{rcs}} \times\left(\mathrm{B}_{\mathrm{j}} \int_{\partial \widehat{D}} \widehat{\boldsymbol{w}}(\hat{\boldsymbol{x}}) e^{i k \gamma_{\mathrm{j}} \hat{\boldsymbol{x}} \cdot \mathrm{B}_{\mathrm{j}}^{T} \widetilde{\boldsymbol{d}}_{\mathrm{rcs}}} d s(\hat{\boldsymbol{x}}) \times \widetilde{\boldsymbol{d}}_{\mathrm{rcs}}\right)
\end{aligned}
$$

Using the EIM-interpolant (5.25) in (5.34) we obtain an affine decomposition approximation to the RCS, for any required accuracy, as

$$
\widehat{\boldsymbol{E}}_{\infty}^{\mathrm{j}}\left[\widehat{\boldsymbol{w}} ; \widetilde{\boldsymbol{d}}_{\mathrm{rcs}}, \boldsymbol{\mu}\right] \approx \widehat{\boldsymbol{E}}_{\infty, M_{\mathcal{F}}}^{\mathrm{j}}\left[\widehat{\boldsymbol{w}} ; \widetilde{\boldsymbol{d}}_{\mathrm{rcs}}, \boldsymbol{\mu}\right]=\sum_{m=1}^{M_{\mathcal{F}}} \boldsymbol{\theta}_{\infty, m}^{\mathrm{j}}\left(\boldsymbol{\mu}, \widetilde{\boldsymbol{d}}_{\mathrm{rcs}}\right) \times \widehat{\mathbf{f}}[\widehat{\boldsymbol{w}} ; m] \times \mathrm{B}_{\mathrm{j}}^{T} \widetilde{\boldsymbol{d}}_{\mathrm{rcs}},
$$

where $\widehat{\mathbf{f}}$ is given by (5.29) and

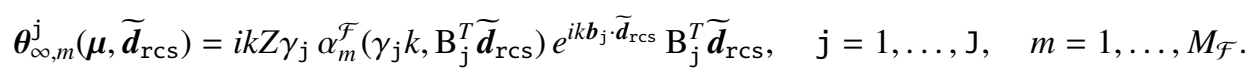

\subsection{Summary and computational cost}

Through judicious choice of various parameter dependent functions in the electromagnetic scattering problem and their EIM-interpolants, we have demonstrated that two/four-dimensional surface integrals in multiple scattering interactive/individual sesquilinear forms and input/output operators can be, approximately, reduced to the evaluation of integrals only on the surface of the reference obstacle $\widehat{D}$ with associated integrands independent of (i) the number of scatteres, shapes, and locations; and (ii) all parameters in the large parameter space governing the parameterized electromagnetic scattering problem.

Let us summarize the developments of the previous sections and provide an overview of the algorithm and its computational cost.

Offline procedure: In the Offline procedure, a reduced basis $\widehat{\mathbb{V}}_{N}$ is assembled for the reference shape $\partial \widehat{D}$ following the lines of Section 4.1. It is worth noticing that the affine decompositions developed therein, based on the EIM as described in Section 5.2 and 5.3, can be reused for assembling the mass matrices and right hand sides in the problem with multiple scatterers. Indeed, given the basis $\left\{\widehat{\boldsymbol{\xi}}_{r}\right\}_{r=1}^{N}$ of the reduced basis space $\widehat{\mathbb{V}}_{N}$, we can use the precomputed matrices associated to the parameter independent forms

$$
\left.\left.\widehat{\left[\mathrm{A}_{1}^{m}\right.}\right]_{r, s}=\widehat{a 1}\left[\widehat{\boldsymbol{\xi}}_{r}, \widehat{\boldsymbol{\xi}}_{s} ; m\right], \quad \widehat{\left[\mathrm{A}_{2}^{m}\right.}\right]_{r, s}=\widehat{a 2}\left[\widehat{\boldsymbol{\xi}}_{r}, \widehat{\boldsymbol{\xi}}_{s} ; m\right], \quad r, s=1, \ldots, N, \quad m=0, \ldots, M_{\mathcal{M}}
$$

and the following three dimensional vectors

$$
\widehat{\mathbf{F}}_{s}^{m}=\widehat{\mathbf{f}}\left[\widehat{\boldsymbol{\xi}}_{s} ; m\right], \quad s=1, \ldots, N, \quad m=1, \ldots, M_{\mathcal{F}}
$$


Further, following the lines of Section 5.1, an EIM is required to get $M_{\mathcal{G}}$ EIM-sample points $\left\{k^{m}, \gamma^{m}, \mathrm{~B}^{m}, \boldsymbol{c}^{m}\right\}_{m=1}^{M_{\mathcal{G}}} \in \mathcal{D}_{1}$ which allows to precompute the reflection matrices

$$
\widehat{\mathrm{R}}_{t, \bar{t}}^{m}=\left[\widehat{R 1}\left[\widehat{\boldsymbol{\xi}}_{r}, \widehat{\boldsymbol{\xi}}_{s} ; m\right]\right]_{t, \bar{t}}, \quad \widehat{\mathrm{R}}_{t, \bar{t}}^{m}=\widehat{R 2}\left[\widehat{\boldsymbol{\xi}}_{r}, \widehat{\boldsymbol{\xi}}_{s} ; m\right], \quad r, s=1, \ldots, N, \quad m=1, \ldots, M_{\mathcal{G}} .
$$

Online procedure: For any new parameter value, for the first iteration (4.6) $(\ell=1)$ of the reduced basis iterative scheme, the impact on each obstacle is chosen to be only from the initial incident wave $\boldsymbol{E}^{\text {inc }}$. Hence using (5.19)-(5.21) and (5.28), we solve J uncoupled (parallel) $N$ dimensional linear systems

$$
\widehat{a}_{M_{\mathcal{M}}}^{\mathrm{ii}}\left[\widehat{\boldsymbol{w}}_{N, \mathrm{i}}^{(1)} \widehat{\boldsymbol{v}}_{N} ; \boldsymbol{\mu}\right]=\widehat{f}_{M_{\mathcal{F}}}^{\mathrm{i}}\left[\widehat{\boldsymbol{v}}_{N} ; \boldsymbol{\mu}\right], \quad \forall \widehat{\boldsymbol{v}}_{N} \in \widehat{\mathbb{V}}_{N}, \quad \mathrm{i}=1, \ldots, \mathrm{J} .
$$

Using (5.19) and (5.28), we are required to set up and solve the associated $N$ dimensional system

$$
\mathrm{A}_{\mathrm{i}} \widehat{\mathrm{W}}_{\mathrm{i}}^{(1)}=-\sum_{m=1}^{M_{\mathcal{F}}} \gamma_{\mathrm{i}} e^{i k \boldsymbol{b}_{\mathrm{i}} \cdot \widetilde{\boldsymbol{d}}_{\mathrm{inc}}} \alpha_{m}^{\mathcal{F}}\left(\gamma_{\mathrm{i}} k, \mathrm{~B}_{\mathrm{i}}^{T} \widetilde{\boldsymbol{d}}_{\mathrm{inc}}\right) \mathbf{F}^{m, \mathrm{i}}, \quad \mathrm{i}=1, \ldots, J,
$$

where

$$
\mathrm{A}_{\mathrm{i}}=i k Z \gamma_{\mathrm{i}} \sum_{m=0}^{M_{\mathcal{M}}} \alpha_{m}^{\mathcal{M}}\left(k \gamma_{\mathrm{i}}\right)\left\{\widehat{\mathrm{A}_{1}^{m}}-\frac{1}{k^{2} \gamma_{\mathrm{i}}^{2}} \widehat{\mathrm{A}_{2}^{m}}\right\}, \quad\left[\mathbf{F}^{m, \mathrm{i}}\right]_{s}=\mathrm{B}_{\mathrm{i}}^{T} \boldsymbol{p}_{\text {inc }} \cdot \widehat{\mathbf{F}}_{s}^{m} \quad n=1, \ldots, N .
$$

In (5.41)-(5.42), for each $\mathrm{i}=1, \ldots, \mathrm{J}$, setting up the coefficients $\alpha_{m}^{\mathcal{F}}\left(\gamma_{\mathrm{i}} k, \mathrm{~B}_{\mathrm{i}}^{T} \widetilde{\boldsymbol{d}}_{\text {inc }}\right)$ and $\alpha_{m}^{\mathcal{M}}\left(k \gamma_{\mathrm{i}}\right)$, respectively, requires the solution of $M_{\mathcal{F}}$ and $M_{\mathcal{M}}$ dimensional lower triangular linear systems, using the EIM-based interpolation matrices.

The subsequent iterations (4.7) are computed by incorporating reflections from other obstacles and solving the uncoupled systems (using the affine decomposition (5.12)), for each $i=1, \ldots, \mathrm{J}$ :

$$
\widehat{a}_{M_{\mathcal{M}}}^{\mathrm{ii}}\left[\widehat{\boldsymbol{w}}_{N, \mathrm{i}}^{(\ell+1)} \widehat{\boldsymbol{v}}_{N} ; \boldsymbol{\mu}\right]=-\sum_{\substack{j=1 \\ j \neq i}}^{\mathrm{J}} \widehat{a}_{M_{\mathcal{G}}}^{\mathrm{ij}}\left[\widehat{\boldsymbol{w}}_{N, \mathrm{i}}^{(\ell)}, \widehat{\boldsymbol{v}}_{N} ; \boldsymbol{\mu}\right], \quad \forall \widehat{\boldsymbol{v}}_{N} \in \widehat{\mathbb{V}}_{N}, \quad \ell=1, \ldots, L-1 .
$$

That means that for each $\ell=1, \ldots, L-1$, having computed $\widehat{\mathrm{w}}_{\mathrm{N}}^{(\ell)}$ (by solving $\mathrm{J}$ uncoupled $N$ dimensional linear systems), we compute $\widehat{\mathrm{w}}_{\mathrm{N}}^{(\ell+1)}$ by solving similar $N$ dimensional systems with stiffness matrix $\mathrm{A}_{\mathrm{i}}$ corresponds to the ith particle in the configuration and the source term corresponds to the reflected interactive waves from other particles impinging on the $i$-th particle obstacle, for $i=1, \ldots, \mathrm{J}$ :

$$
\mathrm{A}_{i} \widehat{\mathrm{w}}_{\mathrm{i}}^{(\ell+1)}=-i k Z \sum_{\substack{j=1 \\ j \neq i}}^{\mathrm{J}} \gamma_{\mathrm{j}} \sum_{m=1}^{M_{\mathcal{G}}} \alpha_{m}^{\mathcal{G}}\left(k \gamma_{\mathrm{i}}, \gamma_{\mathrm{ij}}, \mathrm{B}_{\mathrm{ij}}, \boldsymbol{c}_{\mathrm{ij}}\right)\left[\mathrm{R}_{\mathrm{ij}}^{m} \widehat{\mathrm{w}}_{\mathrm{i}}^{(\ell)}\right], \quad \ell=1, \ldots, L-1,
$$

where, for $m=1, \ldots, M_{\mathcal{G}}$, the $N \times N$ reflection matrix $\mathrm{R}_{\mathrm{ij}}^{m}$ can be easily setup using (5.12) and from the stored offline matrices in (5.39) as

$$
\mathrm{R}_{\mathrm{ij}}^{m}=\sum_{t, \bar{t}=1}^{3}\left(\mathrm{~B}_{\mathrm{j}}^{T} \mathrm{~B}_{\mathrm{i}}\right)_{t, \bar{t}} \widehat{\mathrm{R}}_{t, \bar{t}}^{m}-\frac{1}{k^{2} \gamma_{\mathrm{i}} \gamma_{\mathrm{j}}} \widehat{\mathrm{R}}^{m}, \quad \mathrm{i}, \mathrm{j}=1, \ldots, \mathrm{J}, \quad \mathrm{i} \neq \mathrm{j}
$$

We note that setting up $\alpha_{m}^{\mathcal{G}}\left(k \gamma_{i}, \gamma_{i j}, B_{i j}, c_{i j}\right)$ in (5.44) for each $i, j=1, \ldots, j, i \neq j$ requires the solution of a EIM-based $M_{\mathcal{G}}$ dimensional triangular linear system.

Recall that having computed the solution vector $\widehat{\mathrm{w}}_{i}^{(\mathrm{L})}$ of $(5.44)$, we compute the reduced basis approximation to the surface current in (2.9)-(2.10) for any $\boldsymbol{\mu} \in \mathcal{D}$, as

$$
\boldsymbol{w}_{N, L}(\boldsymbol{x} ; \boldsymbol{\mu})=\sum_{\mathrm{i}=1}^{\mathrm{J}}\left(\widehat{\mathcal{P}}_{\mathrm{i}}^{-1} \widehat{\boldsymbol{w}}_{\mathrm{i}}^{L}\right)(\boldsymbol{x} ; \boldsymbol{\mu}), \quad \boldsymbol{x} \in \partial \Omega=\bigcup_{\mathrm{j}=1}^{\mathrm{J}} \partial \Omega_{\mathrm{j}}
$$


Finally, the RCS of the configuration for any parameter $\widetilde{\boldsymbol{d}}_{\mathrm{rcs}}$, using (5.46) with minimal computational effort can be computed. We recall (5.32)-(5.36) and (5.29) and compute the online approximation to the RCS of the configuration using

$$
\operatorname{RCS}\left[\boldsymbol{w}_{N, L} ; \widetilde{\boldsymbol{d}}_{\mathrm{rcs}}, \boldsymbol{\mu}\right]=10 \log _{10}\left(4 \pi \frac{\left|\boldsymbol{E}_{\infty, M_{\mathcal{F}}}\left[\boldsymbol{w}_{N, L} ; \widetilde{\boldsymbol{d}}_{\mathrm{rcs}}, \boldsymbol{\mu}\right]\right|^{2}}{\left|\boldsymbol{p}_{\mathrm{inc}}\right|^{2}}\right),
$$

where

$$
\widehat{\boldsymbol{E}}_{\infty, M_{\mathcal{F}}}\left[\boldsymbol{w}_{N, L} ; \widetilde{\boldsymbol{d}}_{\mathrm{rcs}}, \boldsymbol{\mu}\right]=\sum_{\mathrm{j}=1}^{J} \sum_{n=1}^{N} \sum_{m=1}^{M_{\mathcal{F}}} \widehat{\mathrm{w}}_{\mathrm{s}, \mathrm{j}}^{(\mathrm{L})} \boldsymbol{\theta}_{\infty, m}^{\mathrm{j}}\left(\boldsymbol{\mu}, \widetilde{\boldsymbol{d}}_{\mathrm{rcs}}\right) \times\left[\mathrm{B}_{\mathrm{j}} \widehat{\mathbf{F}}_{n}^{m}\right] \times \mathrm{B}_{\mathrm{j}}^{T} \widetilde{\boldsymbol{d}}_{\mathrm{rcs}},
$$

with setting up $\boldsymbol{\theta}_{\infty, m}^{\mathrm{j}}\left(\boldsymbol{\mu}, \widetilde{\boldsymbol{d}}_{\mathrm{rcs}}\right)$, given by (5.36), requires solution of a EIM-based $M_{\mathcal{F}}$ dimensional triangular linear system for each $i, j=1, \ldots, \mathrm{J}, i \neq j$, and $\widehat{\mathbf{F}}_{n}^{m}$ being computed offline as described in (5.38).

The inexpensive online computation is dominated only by solutions of smaller linear systems and can be summarized as:

- Solutions of $\mathrm{J}$ uncoupled $\left(M_{\mathcal{M}}+M_{\mathcal{F}}\right)$ lower triangular systems to set up (5.41).

- Solutions of J uncoupled $N$ dimensional dense linear systems (5.41)

- Solutions of $\mathrm{J}^{2}-\mathrm{J}$ uncoupled $M_{\mathcal{G}}$ lower triangular systems to set up (5.44).

- Solutions of J uncoupled $N$ dimensional dense linear systems (5.44).

- Solutions of $\mathrm{J}$ uncoupled $M_{\mathcal{F}}$ lower triangular systems to setup (5.48).

All the above system setup and solves can be easily parallelized, with minimal communication, with respect to the EIM sample points and the number of scatterers in the configuration.

\section{Numerical experiments}

We seek to validate the offline/online iterative reduced basis algorithm for several multiple scatterer configurations associated with the parameterized electromagnetic scattering model problem. The reference obstacle $\widehat{D}$ for the configurations are a metallic unit sphere or a metallic cavity, both of unit length. For the boundary element truth solution, we take ten points per wavelength discretization in each dimension and use the Cerfacs Electromagnetic Solver Code (CESC) to guarantee numerically good reference solutions.

The sphere as a reference obstacle is useful to validate against a well known benchmark dynamic configuration of two metallic spheres for which experimentally measured RCS values are known [30] for a few frequencies. Parameters for the benchmark dynamic configurations include varying distance between the spheres. The parameter domain for all our offline simulations in this section, includes varying incident directions and associated vertical and horizontal unit polarization vectors. Using spherical coordinates, the online incident direction choice is represented by the pair $\left(\theta_{\text {inc }}, \phi_{\text {inc }}\right)$ as

$$
\widetilde{\boldsymbol{d}}_{\text {inc }}=-\left(\sin \theta_{\text {inc }} \cos \phi_{\text {inc }}, \sin \theta_{\text {inc }} \sin \phi_{\text {inc }}, \cos \theta_{\text {inc }}\right)^{T}, \quad \theta_{\text {inc }} \in[0, \pi], \quad \phi_{\text {inc }} \in[0,2 \pi),
$$

and the online incident direction is vertically polarized (that is, $\boldsymbol{p}_{\text {inc }}=\frac{\partial}{\partial \theta_{\text {inc }}} \widetilde{\boldsymbol{d}}_{\text {inc }}$ ). The online receiver direction $\left(\theta_{\mathrm{rcs}}, \phi_{\mathrm{rcs}}\right)$ for the RCS calculation is represented as

$$
\widetilde{\boldsymbol{d}}_{\mathrm{rcs}}=\left(\sin \theta_{\mathrm{rcs}} \cos \phi_{\mathrm{rcs}}, \sin \theta_{\mathrm{rcs}} \sin \phi_{\mathrm{rcs}}, \cos \theta_{\mathrm{rcs}}\right)^{T}, \quad \theta_{\mathrm{inc}} \in[0, \pi], \quad \phi_{\mathrm{inc}} \in[0,2 \pi),
$$

so that $\left(\theta_{\text {inc }}, \phi_{\text {inc }}\right)=\left(\theta_{\text {rcs }}, \phi_{\text {rcs }}\right)$ corresponds to the backscattering (monostatic) RCS. The minimal physical distance between particles (measured between centers) in a configuration is denoted by $d$ and hence the minimal frequency based distance between obstacles is $k d$.

Parameters required in the multiple electromagnetic scattering process to obtain results for all plots given in this section are chosen as part of the online process described in Section 4.2, after construction of the associated reduced bases through the offline process in Section 4.1. We demonstrate substantial reduction (even by over 90\%, see Table 1) 
in the dimension $N$ of the reduced basis compared to the dimension $\mathcal{N}$ of the truth boundary element space and yet achieve good accuracy.

Example 1. (Two metallic unit spheres with two fixed frequencies but varying seperation.)

We consider two experimental results given [30, Figure 3] for an endfire configuration consisting of two unit metallic unit spheres, for $k=7.41$ and $k=11.048$. For each of these cases, we use the offline algorithm in Section 4.1, with wavenumber fixed (thus $k^{-}=k^{+}$) to construct $N$ snapshot parameters and the reduced basis space $\widehat{\mathbb{V}}_{N}$, corresponding to the reference unit sphere $\widehat{D}$.

In Table 1, we give an an overview of the boundary element mesh $(h)$, dimensions of the truth boundary element space $(\mathcal{N})$, the reduced basis space $(N)$ and the residual error achieved for the assembling of the reduced basis space. As observed in Table 1, the dimension of the reduced basis is only a fraction of that of the boundary element space, demonstrating the inexpensive part of the online computation.

\begin{tabular}{|c|c|c|c|c|}
\hline$k$ & mesh size $(h)$ & Dim BEM $(\mathcal{N})$ & Dim Reduced $(N)$ & Residual error \\
\hline 7.41 & $0.826 \mathrm{e}-1$ & 7680 & 563 & $9.73 \mathrm{e}-4$ \\
\hline 11.048 & $0.551 \mathrm{e}-1$ & 17280 & 918 & $9.94 \mathrm{e}-4$ \\
\hline
\end{tabular}

Table 1: (Example 1.) Accuracy of reduced basis space compared to BEM space

We use the online algorithm in Section 4.2 with $\mathrm{J}=2, \gamma_{\mathrm{i}}=1, B_{\mathrm{i}}=I$ and use varying translation (moving distance) parameters $\boldsymbol{b}_{i}, i=1,2$, with the domain of these parameters dictated by the $k d$ values in [30, Figures 3]. The online computations include a large number of configurations of two metallic spheres. For each such configuration, we compute the backscattered RCS and plot our results, compared with those in [30, Figure 3]. Results in Figure 1, with iterative reduced basis based RCS superimposed on Figures in [30, Figure 3] demonstrate the accuracy of our algorithm compared with the experimental results reported in [30, Figure 3].
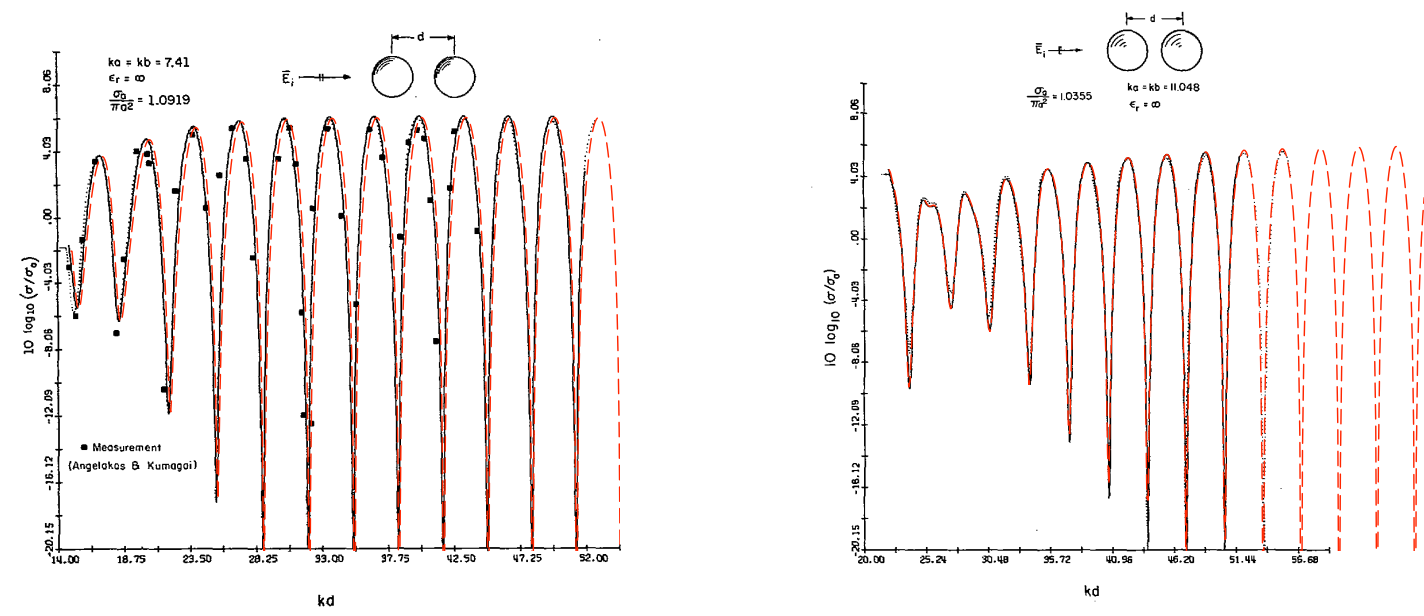

Figure 1: (Example 1.) Comparison with benchmark experimental results in [30, Figure 3] for $k=7.41$ (left) and for $k=11.048$ (right) for parameter values $k d$ measuring the electric distance.

Example 2. (Two metallic unit spheres with variable wavenumbers and distance.)

We extend the parameter domain in the previous example to first consider wavenumbers $k \in[1,3]$, build a reduced basis and then another reduced basis with $k \in[3,5]$. We recall that because of the EFIE formulation, we need to exclude the resonant wavenumbers from the parameter domain for the offline construction of the reduced basis. The 
resonant wavenumbers for the unit sphere are given in [31, Table 6.1] and in the interval [1,5] they are $k_{1}=2.743$, $k_{2}=3.870, k_{3}=4.493$ and $k_{4}=4.973$.

In Table 2, we illustrate the boundary element mesh $(h)$, dimensions of the truth boundary element space $(\mathcal{N})$ and reduced basis space $(N)$ and the residual error achieved for the reduced basis space that include two variable wavenumber intervals. The online computations include a large number of configurations of two metallic spheres.

\begin{tabular}{|c|c|c|c|c|}
\hline$k \in$ & mesh size $(h)$ & Dim BEM $(\mathcal{N})$ & Dim Reduced $(N)$ & Residual error \\
\hline$[1,3]$ & 0.110 & 4320 & 290 & $2.23 \mathrm{e}-4$ \\
\hline$[3,5]$ & 0.110 & 4320 & 509 & $2.64 \mathrm{e}-4$ \\
\hline
\end{tabular}

Table 2: (Example 2.) Accuracy of reduced basis space compared to BEM space.

We analyze the error between the reduced basis approximation and the truth solution with respect to the frequency based minimal distance $k d$ for several snapshot of the configuration. The error is taken over the parameter set of all incident angles with $\theta_{\text {inc }}=\pi / 2$ and 100 samples points distributed equally for $\phi_{\text {inc }} \in[0, \pi / 2]$ and this includes both the broadside and endfire incidence.

In Figure 2, we show the $L_{2}$-norm relative error of the reduced basis approximation $\boldsymbol{w}_{N}(\boldsymbol{\mu})$ with respect to the truth solution $\boldsymbol{w}_{h}(\boldsymbol{\mu})$ depending on the electric distance between the two objects, for the six wavenumbers $k=1,2,3,4,5,6$. Figure 2 demonstrates low to high-order accuracy of the reduced basis approximation as the electric distance between the moving metallic spheres increases. The corresponding total number of iterations (denoted by $L$ in Section 4.2 , with convergence assumed at tol $=10^{-8}$ ) is presented in Figure 3 . The number of iterations decreases algebraically as the distance of the two moving spheres increases but remain very small even for the case of the spheres being close.

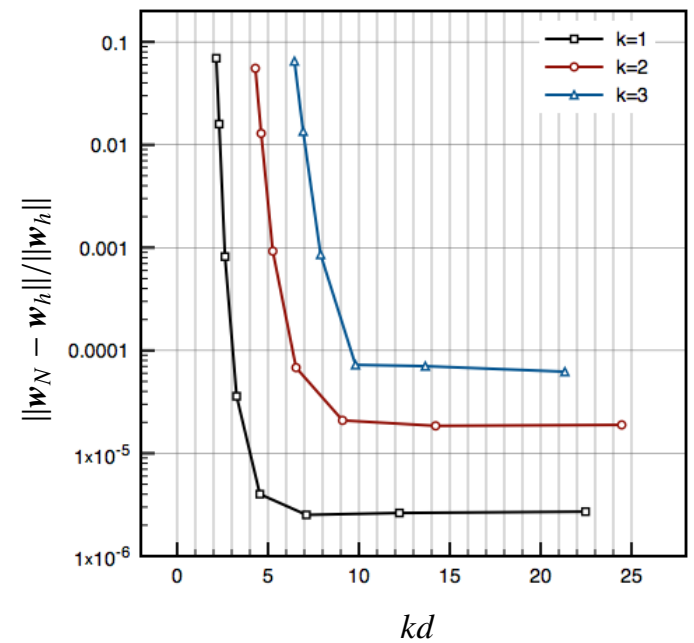

(a)

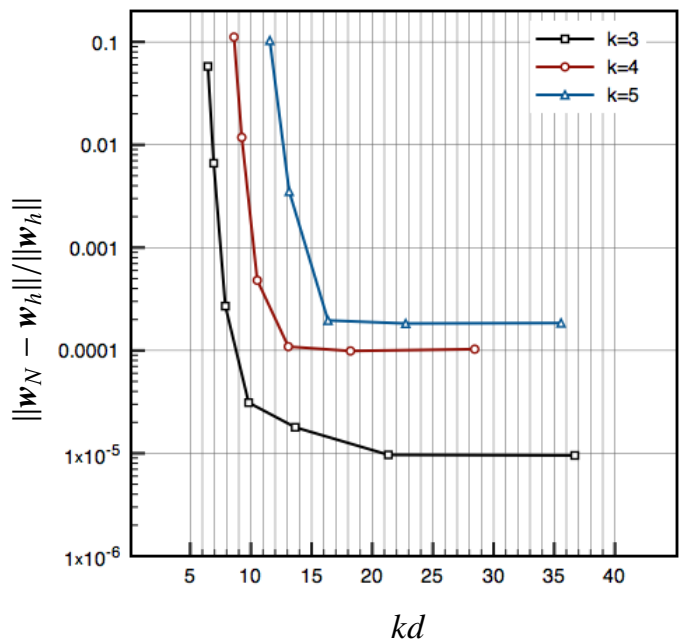

(b)

Figure 2: (Example 2.) The relative error of the reduced basis approximation with respect to the truth solution as a function of the frequency based distance $k d$ between two metallic unit spheres for six online choices of the wavenumber.

\section{Example 3. (Two metallic spheres with variable electric size parameters.)}

The parameter domain for this simulation includes a shrink parameter $(\gamma)$ interval of $[0.5,1]$. The online multiple scattering configuration consists of two spheres that are scaled and translated versions of the reference unit sphere, with $k=3$, separated by a fixed physical distance $d=4$, and angle of incidence $\theta_{\text {inc }}=\phi_{\text {inc }}=0$.

For the online iterative reduced basis computation, we choose two arbitrary pair of parameters, $\left(\gamma_{1}, \gamma_{2}\right)=(0.865,0.691)$ and $\left(\gamma_{1}, \gamma_{2}\right)=(0.549,0.982)$, and compute the RCS of the resulting configuration for $\phi_{\mathrm{rcs}}=0$ and varying $\theta_{\mathrm{rcs}} \in$ $\left[0, \frac{\pi}{2}\right]$. To check the accuracy of our computation, we also compute the truth RCS using the standard boundary element 


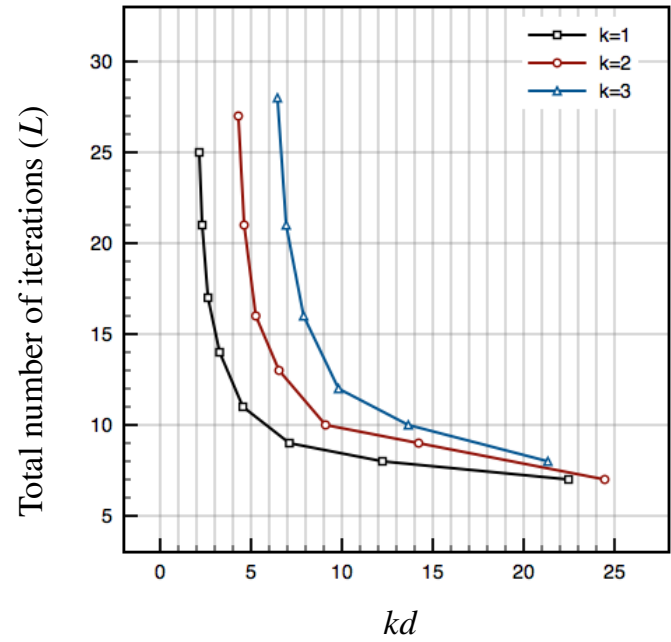

(a)

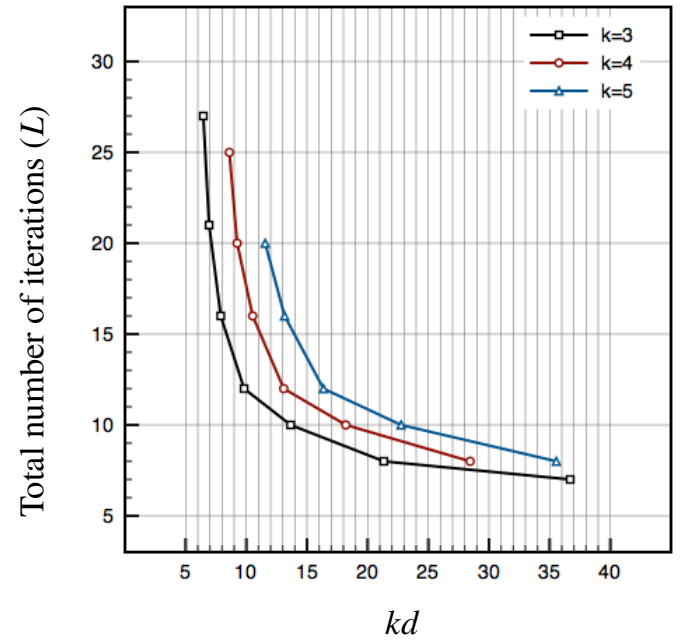

(b)

Figure 3: (Example 2.) The total number of online multiple scattering iterations a function of the frequency based distance $k d$ between two moving metallic unit spheres for six online choice of wavenumber to compute reduced basis approximations.

method directly for the pair of two particle configurations. Comparison between the reduced basis RCS and the truth RCS, illustrated in Figure 4, further demonstrates the accuracy of our efficient offline/online iterative reduced basis algorithm. Next we compute the backscattering $\left(\theta_{\mathrm{rcs}}=\phi_{\mathrm{rcs}}=0\right) \operatorname{RCS}$ for large number of parameters $\gamma_{1}, \gamma_{2} \in[0.5,1]$ and plot the RCS as a function of $\gamma_{1}, \gamma_{2}$ and finally compute the RCS with varying $\gamma_{1} \in[0.5,1]$ and $\gamma_{2}=1.5-\gamma_{1}$ and plot in the RCS as a function of $\theta_{\mathrm{rcs}} \in\left[0, \frac{\pi}{2}\right]$ and $\gamma_{1}$. These results are in Figure 5.

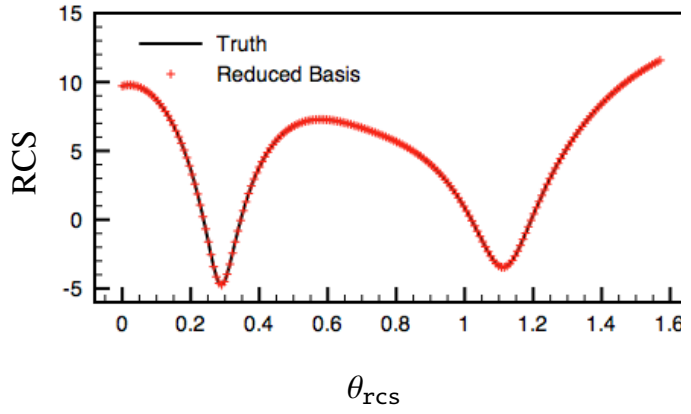

(a)

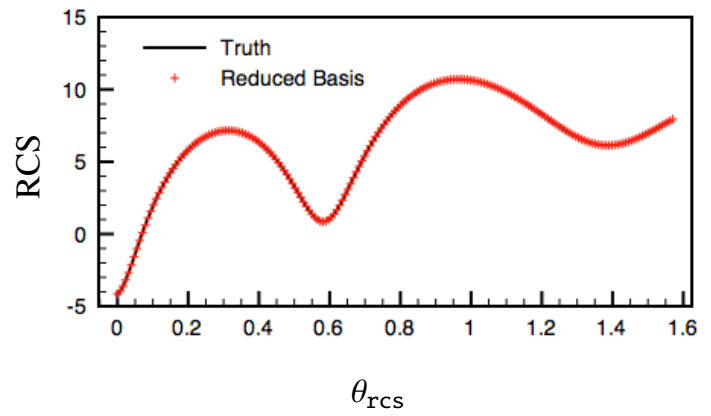

(b)

Figure 4: $\left(\right.$ Example 3.) Validation of the reduced basis RCS for $\left(\gamma_{1}, \gamma_{2}\right)=(0.865,0.691)($ left $)$ and $\left(\gamma_{1}, \gamma_{2}\right)=(0.549,0.982)($ right $)$.

\section{Example 4. (Metallic spheres with varying wavenumbers, sources and observations.)}

Having demonstrated the power of our reduced basis algorithms for two sphre configurations, we use the same reduced basis constructed offline for the reference unit sphere in the first three examples to simulate the RCS of a $6 \times 6$ lattice configuration with 36 metallic spheres, illustrated in Figure 6 (with $d=6$ ), for wavenumbers $k \in[1,5]$ and for tens of thousands of incident and observed directions. In Figure 7 we plot the RCS, with variable observation in the direction $\theta_{\mathrm{rcs}}=\frac{\pi}{2}, \phi_{\mathrm{rcs}} \in[0,2 \pi]$ for different wavenumbers $k=1,2,4,5$ and varying incident directions $\phi_{\text {inc }} \in[0,2 \pi]$ (and $\left.\theta_{\text {inc }}=\frac{\pi}{2}\right)$ of the plane electromagnetic wave that illuminates the target. The simulated values in Figure 7 demonstrate strong signals when the receiver is located opposite to the transmitter (that is, $\phi_{\mathrm{rcs}}=\phi_{\text {inc }}+\pi$ ). 


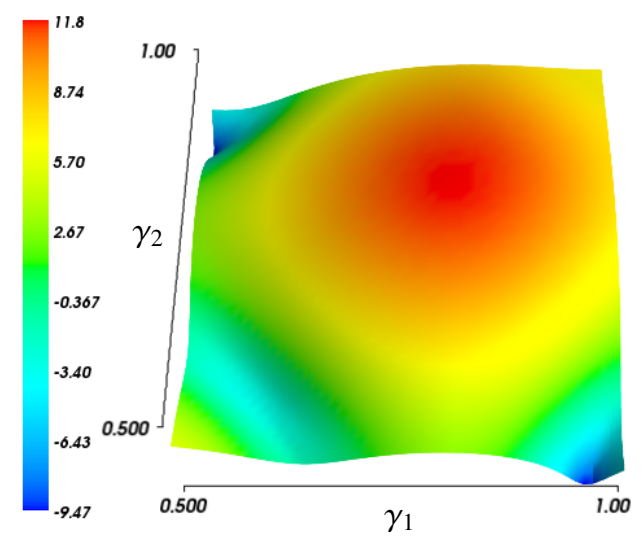

(a) RCS of configurations as a function of sphere size parameters $\gamma_{1}, \gamma_{2} \in[0.5,1.0]$ for a fixed observation point $\theta_{\mathrm{rcs}}=\phi_{\mathrm{rcs}}=0$.

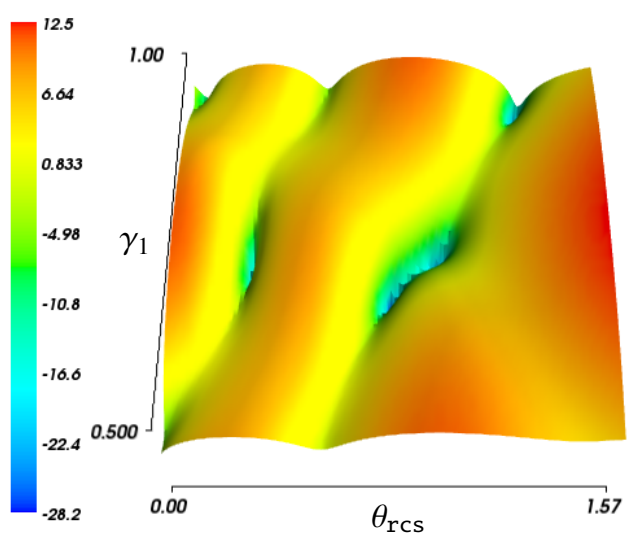

(b) RCS of configurations as a function of a sphere size parameter $\gamma_{1} \in[0.5,1.0]$, for variable observation directions $\theta_{\mathrm{rcs}} \in\left[0, \frac{\pi}{2}\right]$, with $\gamma_{2}=1.5-\gamma_{1}$ and $\phi_{\mathrm{rcs}}=0$.

Figure 5: (Example 3.) RCS of configurations with varying sphere size for a fixed (left) and variable receiver directions (right).

For varying wavenumbers $k \in[1,4.972]$ the RCS, measured in the direction $\theta_{\mathrm{rcs}}=\frac{\pi}{2}, \phi_{\mathrm{rcs}} \in[0,2 \pi]$, is illustrated in Figure 8 with $\theta_{\text {inc }}=0$ and (a) $\theta_{\text {inc }}=\frac{\pi}{2}$ and (b) $\phi_{\text {inc }}=0$ of the incident plane wave. We recall that there are three resonant wavenumbers in $[1,4.972]$ for the reference spheres, namely $k_{1}=2.743, k_{2}=3.870, k_{3}=4.493$ and the reduced basis parameter domain does not include these numbers. Hence, as expected, the iterative reduced basis scheme does not converge for values near $k_{1}, k_{2}, k_{3}$, as observed through the gaps occurring in three place in Figure 8. The fourth resonant wavenumber in $[1,5]$ is $k_{4}=4.973$ and we observed similar property around $k_{4}$.

\section{Example 5. (Metallic open cavities with varying rotations.)}

The initial electromagnetic scattering consists of two metallic open cavities $\Omega_{1}, \Omega_{2}$, situation along the $y$-axis separated by a physical distance $d=4$, with variability determined by rotating $\Omega_{1}$ by a clockwise angle $\alpha_{1}$ around the $z$-axis and $\Omega_{2}$ by an anti-clockwise $\alpha_{2}$ around the $z$-axis, as illustrated in Figure 9. The electromagnetic scattering configuration is tested using incident waves with varying wavenumbers $k \in[1,3]$, incident angles, and in the first case with both boxes rotated by same angles $\left(\alpha_{1}=\alpha_{2}\right)$ in $\left[0, \frac{\pi}{2}\right]$.

For $\alpha_{1}=\alpha_{2}=0$, the two boxes are exact copies of the reference shape $\widehat{D}$ which is an open cavity of unit length centered at the origin and open towards the positive x-axis. For $\alpha_{1}=\alpha_{2}=\frac{\pi}{2}$, their openings are facing each other. Details of offline assembly and accuracy of the reduced basis for the reference open box are given in Table 3 . In order

\begin{tabular}{|c|c|c|c|c|}
\hline$k \in$ & mesh size $(h)$ & Dim BEM $(\mathcal{N})$ & Dim Reduced $(N)$ & Residual error \\
\hline$[1,3]$ & 0.117 & 1795 & 239 & $7.144 \mathrm{e}-4$ \\
\hline
\end{tabular}

Table 3: (Example 5.) Accuracy of reduced basis space for the open cavity compared to BEM space.

to check the accuracy of the online iterative reduced basis multiple particle algorithm, we choose two pairs of random parameters, $\left(k, \alpha_{1}\right)=(1.395,0.7552)$ and $\left(k, \alpha_{1}\right)=(2.491,1.3774)$ and choose the incident wave direction as $\theta_{\text {inc }}=\frac{\pi}{2}$, $\phi_{\text {inc }}=0$. The nature of the random test parameters is that no values of the training sets of any sampling process such as the EIM or the reference scatterer reduced basis method is chosen, i.e., the computed results rely entirely on the accuracy of the reduced basis and the iterative procedure. We compare the RCS (with $\theta_{\text {rcs }}=\frac{\pi}{2}$ and $\phi_{\text {rcs }} \in[0,2 \pi]$ ) of the reduced basis solution with the RCS of the truth approximation. Results in Figure 10(a) demonstrate the accuracy of the RCS obtained using the reduced basis online approximation.

Next we consider the case in which each of the two boxes are rotated individually. The first box $\Omega_{1}$ is rotated by an angle $\alpha_{1} \in\left[\frac{\pi}{4}, \frac{\pi}{2}\right]$ and $\Omega_{2}$ is rotated by an angle $\alpha_{2} \in\left[\frac{\pi}{4}, \frac{\pi}{2}\right]$, for a fixed wavenumber $k=2.76$. To check the accuracy of the reduced basis RCS, we choose a random pair of rotation parameter values $\alpha_{1}=1.5396, \alpha_{2}=1.1299$ 

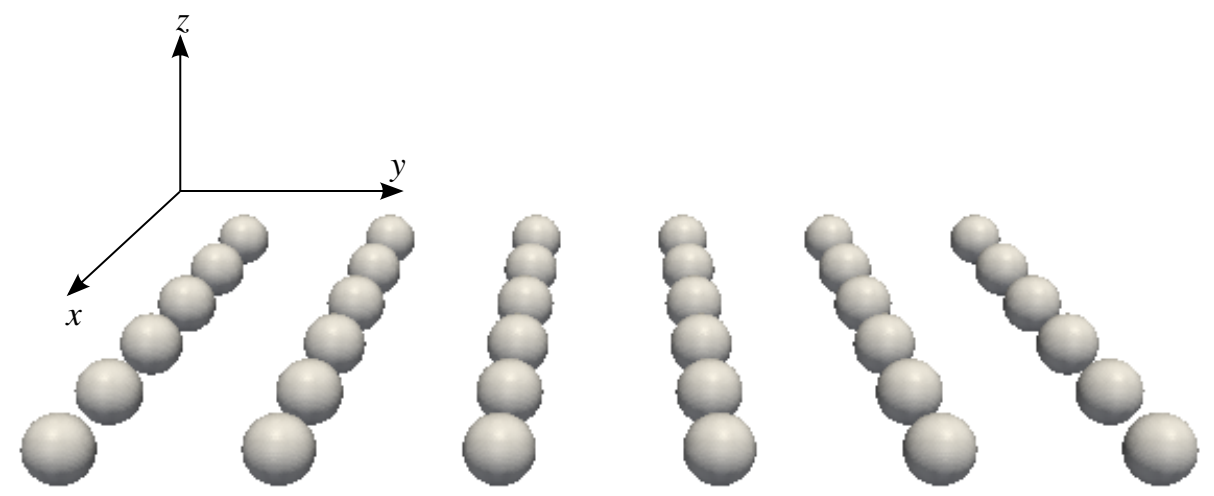

Figure 6: (Example 4.) A multiple scattering configuration with 36 metallic spheres.

and demonstrate the accuracy of the reduced basis RCS in Figure 10(b) as compared to the truth approximation.

In Figure 11(a), we plot the monostatic RCS as a function of varying wavenumber $k \in[1,3]$ and the varying rotation parameter $\alpha_{1}=\alpha_{2} \in\left[0, \frac{\pi}{2}\right]$ for a fixed $\theta_{\text {inc }}=\theta_{\text {rcs }}=\frac{\pi}{2}, \phi_{\text {inc }}=\phi_{\text {rcs }}=0$. We observed that for $k=2.76$, there is an angle $\alpha=54 . \overline{54}$ where there is a significant reduction in the backscatter. In Figure 11(b), for $k=2.76$, we plot the monostatic RCS as a function of the two individually rotated angles and observed almost no backscattering for all angles satisfying approximatively $\alpha_{1}+\alpha_{2}=109 . \overline{09}$. This serves an examples of the kind of online optimization and parameter studies made available with the methods discussed in this work.

\section{Example 6. (An uncertain configuration with 9 metallic open cavities)}

Our approach of first establishing a (offline computation based) back-end solver to facilitate fast (online) simulation of the front-end forward model enables us to carry out sensitivity analyses for problems where some parameters are unknown or only known in a probabilistic sense. This offers a valuable approach to, among other things, quantify the impact of uncertainties on the output of interest.

Although the characterization of the impact of uncertainties on the RCS for the full set of model parameters is beyond the scope of this work, we illustrate below the modeling of the expected value $(E)$, variance $(V)$, and associated interval $[E-\sqrt{V}, E+\sqrt{V}]$ of the RCS when one of the parameters in the electromagnetic scattering configuration is assumed to be specified by a continuous random variable. We consider a scatterer configuration consisting of 9 open metallic cavities in a $3 \times 3$ grid (with open end facing towards the positive $x$-axis) in the $y z$-plane separated by a physical distance $d=3$. The $z$-coordinate of the box in the middle [whose center has coordinates $(x, y, z)=(0,3,3)$ ] is perturbed by a continuous uniformly distributed random variable $\delta \in[-1,1]$, see Figure 12 .

We fix the wavenumber to be $k=3$ and carry out the sensitivity analysis for four different angles $\left(\theta_{\text {inc }}=0, \frac{\pi}{6}, \frac{\pi}{3}, \frac{\pi}{2}\right)$ of the incident plane wave with $\phi_{\text {inc }}=0$, i.e., in the the fourth case the incidence is facing the open faces of the boxes from the front. The corresponding statistical results are illustrated in Figure 13 with $\phi_{\mathrm{rcs}}=0$, computed by a direct Monte Carlo approach based on the reduced model.

In Figure 14, where the expected value and variance of the RCS are given for incident electromagnetic waves impinging on the uncertain 9 box configuration with continuous angle of incidence $\theta_{\text {inc }} \in\left[0, \frac{\pi}{2}\right]$.

\section{Example 7. (Configurations with different reference shapes.)}

As a last example we seek to show the versatility and generality of the proposed techniques discussed in the work by illustrating that configurations with two or more reference shapes can be handled within the same framework. In this case, we consider scattering configurations comprised of spheres and open cavities but there is naturally nothing specific about that.

In order to verify accuracy of the basic interactions, we first illustrate in Figure 15 the relative error and number of iterations required for a two body configuration separated by a varying distance $d$ and for wavenumbers $k=1,23$. 
Here, one obstacle is the unit sphere used in Example 2 and the other scattered is the open box introduced in Example 5. The incident field is parametrized by the angles $\theta_{\text {inc }}=\phi_{\text {inc }}=0$. We observe a convergence behavior similar to that of two spheres (Example 2). This is not surprising since the interaction between the obstacles is modeled as linear combination of plane waves, and each reduced basis (for each reference shape) is trained to accurately represent the solution currents. The fact that we are dealing with two different reference shapes does not change this aspect.

Further, we consider two more general configurations of open boxes and unit spheres in a $3 \times 3$-lattice (in the $x y$-plane) separated by a distance $d=4$. However, the $x$ - and $y$-coordinates of the scatterers are randomly perturbed, see Figure 16. The RCS for parameter values $\theta_{\mathrm{rcs}}=\frac{\pi}{2}, \phi_{\mathrm{rcs}} \in[0,2 \pi]$ is plotted in Figure 17 for different incident fields corresponding to $\theta_{\text {inc }}=\frac{\pi}{2}, \phi_{\text {inc }} \in[0,2 \pi]$, in both cases of the unperturbed and perturbed lattice.

\section{Concluding remarks}

The main goal of this work has been to develop a rigorous and carefully validated approach to the efficient and accurate computation of electromagnetic scattering by general scattering configurations consisting of a collection of individual and separated scatterers. This is achieved by combining the recently developed reduced basis methods for parametrized integral equations, in this case the electric field integral equation, for the individual scattering and mutual interaction with a rapid online iterative process to recover the scattering signature from the global configuration. It is noteworthy that in this approach, we never attempt to solve the global problem but rather is required only to develop fast and accurate ways of representing the scattering by reference objects and their mutual interaction.

Although a number of both technical and practical challenges had to be addresses to achieve this, the examples illustrate both the accuracy and generality of the proposed technique and a number of interesting applications areas within design, optimization, and uncertainty quantification has been illustrated. It is also worth emphasizing that the emphasis on electromagnetics and the electric field integral equation serves as an example only and other integral formulation from acoustics or electrostatics can be addressed in a similar fashion, possibly with substantial simplifications.

The most natural limitation of the proposed approach to the computation of scattering by a collection of scatterers is the limitation on how close two individual scatterers can be without severely impacting the accuracy. The source of this limitation is found in the plane wave assumption used to account for the mutual interaction. Once the scatterers get very close, the plane waves do no longer offer a good approximation to the near fields and higher order terms involving dipoles and quadrupoles would be required. However, the computational results suggest that this does not become a serious problem until subwavelength distances and only when high accuracy is required. In such cases, one will be have to account of scatterers in close proximity as a single scatterer.

While we have successfully demonstrated the potential for the proposed combination of reduced basis methods for both single scatterers and their mutual interactions, several challenges and extensions remain. Naturally, extensions to integral formulations of a more robust character such as the combined field integral equation and for volume problems with homogeneous material properties are a natural and should be possible with only minor changes of what is already laid out here. A more central challenge is found the observation that the number of online iterations generally scales with the wavenumber. As this directly impacts the online cost, it is clearly worthwhile considering more advanced iterative techniques. Since the approach proposed here is basically matrix free, it is likely that more robust iterative techniques can be developed.

A final and more substantial challenge lies in the extension of the proposed framework to problems with a very large number of scatterers. This should most naturally be pursued in a fast multi-pole like approach with farfield contributions addressed by a reduced models of clusters of scatterers in which these far field model themselves are resolved using a local iterative reduced basis. We hope to pursue this is near future to enable the rapid online modeling of large parameterized scattering configurations.

\section{Acknowledgement}

The second author acknowledges partial support by OSD/AFOSR FA9550-09-1- 0613. 


\section{References}

[1] M. I. Mishchenko, L. D. Travis, A. A. Lacis, Multiple Scattering of Light by Particles: Radiative Transfer and Coherent Backscattering, Cambridge University Press, 2006.

[2] B. O. Almroth, P. Stern, F. A. Brogan, Automatic choice of global shape functions in structural analysis, AIAA Journal 16 (1978) 525-528.

[3] A. K. Noor, J. M. Peters, Reduced basis technique for nonlinear analysis of structures, AIAA Journal 45 (172) (1980) $487-496$.

[4] A. Barrett, G. Reddien, On the reduced basis method, Z. Angew. Math. Mech. 75 (7) (1995) 543-549.

[5] W. C. Rheinboldt, On the theory and error estimation of the reduced basis method for multi-parameter problems, Nonlinear Anal. 21 (11) (1993) 849-858.

[6] M. D. Gunzburger, Finite element methods for viscous incompressible flows, Computer Science and Scientific Computing, Academic Press Inc., Boston, MA, 1989, a guide to theory, practice, and algorithms.

[7] J. S. Peterson, The reduced basis method for incompressible viscous flow calculations, SIAM Journal on Scientific and Statistical Computing 10 (4) (1989) 777-786. doi:10.1137/0910047.

URL http://link.aip.org/link/?SCE/10/777/1

[8] J. P. Fink, W. C. Rheinboldt, On the error behavior of the reduced basis technique for nonlinear finite element approximations, Z. Angew. Math. Mech. 63 (1) (1983) 21-28.

[9] T. A. Porsching, Estimation of the error in the reduced basis method solution of nonlinear equations, Math. Comp. 45 (172) (1985) $487-496$.

[10] E. Balmes, Parametric families of reduced finite element models: Theory and applications, Machanical Systems and Signal Processing 10 (4) (1996) 381-394.

[11] K. Ito, S. S. Ravindran, A reduced basis method for simulation and control of fluid flows, Journal of Computational Physics 143 (2) (1998) 403-425.

[12] Y. Maday, A. T. Patera, D. V. Rovas, A blackbox reduced-basis output bound method for noncoercive linear problems, in: Nonlinear partial differential equations and their applications. Collège de France Seminar, Vol. XIV (Paris, 1997/1998), Vol. 31 of Stud. Math. Appl., NorthHolland, Amsterdam, 2002, pp. 533-569.

[13] L. Machiels, Y. Maday, I. B. Oliveira, A. T. Patera, D. V. Rovas, Output bounds for reduced-basis approximations of symmetric positive definite eigenvalue problems, C. R. Acad. Sci. Paris Sér. I Math. 331 (2) (2000) 153-158.

[14] K. Veroy, C. Prud'homme, D. Rovas, A.T.Patera, A posteriori error bounds for reduced-basis approximation of parametrized noncoercive and nonlinear elliptic partial differential equations, AIAA.

[15] C. Prud'homme, D. Rovas, K. Veroy, Y. Maday, A. T. Patera, G. Turinici, Reliable real-time solution of parametrized partial differential equations: Reduced-basis output bound methods, Journal of Fluids Engineering 124 (1) (2002) 70-80.

[16] K. Veroy, C. Prud'homme, A. T. Patera, Reduced-basis approximation of the viscous burgers equation: Rigorous a posteriori error bounds, C. R. Acad. Sci. Paris, Série I 337 (9) (2003) 619-624.

[17] M. Barrault, N. C. Nguyen, Y. Maday, A. T. Patera, An "empirical interpolation" method: Application to efficient reduced-basis discretization of partial differential equations, C. R. Acad. Sci. Paris, Série I 339 (2004) 667-672.

[18] M. A. Grepl, Y. Maday, N. C. Nguyen, A. T. Patera, Efficient reduced-basis treatment of nonaffine and nonlinear partial differential equations, Mathematical Modelling and Numerical Analysis.

[19] K. Veroy, Reduced-basis methods applied to problems in elasticity: Analysis and applications, Ph.D. thesis, MIT (June 2003).

[20] G. Rozza, Shape design by optimal flow control and reduced basis techniques: Applications to bypass configurations in haemodynamics, Ph.D. thesis, EPFL

[21] Y. Maday, A. T. Patera, G. Turinici, A priori convergence theory for reduced-basis approximations of single-parameter elliptic partial differential equations, in: Proceedings of the Fifth International Conference on Spectral and High Order Methods (ICOSAHOM-01) (Uppsala), Vol. 17, 2002, pp. 437-446.

[22] B. Fares, J. Hesthaven, Y. Maday, B. Stamm, The reduced basis method for the electric field integral equation, J. Comput. Phys.

[23] M. Ganesh, S. C. Hawkins, A high-order algorithm for multiple electromagnetic scattering in three dimensions, Numer. Algorithms 50 (2009) $469-510$.

[24] J.-C. Nédélec, Acoustic and Electromagnetic Equations, Springer, 2001.

[25] A. Buffa, M. Costabel, D. Sheen, On traces for h (curl, $\mathbf{\Omega})$ in Lipschitz domains, J. Math. Anal. Appl. 276 (2002) 845-867.

[26] A. Buffa, R. Hiptmair, Galerkin boundary element methods for electromagnetic scattering, in: Topics in Computational Wave Propagation Direct and Inverse Problems, Vol. 31 of Lecture Notes in Computer Science and Engineering, Springer, 83-124, 2003, pp. 83-124.

[27] M. Balabane, Boundary decomposition for Helmholtz and Maxwell equations 1: disjoint sub-scatterers, Asymp. Anal. 38 (2004) 1-10.

[28] M. Barrault, Y. Maday, N. C. Nguyen, A. T. Patera, An 'empricial interpolation method': application to efficient reduced-basis discretization of partial differential equations, C. R. Math. Acad. Sci. Paris 339 (2004) 667-672.

[29] Y. Maday, N. C. Nguyen, A. T. Patera, G. S. H. Pau, A general multipurpose interpolation procedure: the magic points, Commun. Pure Appl. Anal. 8 (2009) 383-404.

[30] J. Bruning, Y. Lo, Multiple scattering of em waves by spheres part II-Numerical and experimental results, IEEE Trans. Antennas and Propag. 19 (3) (1971) $391-400$.

[31] J. H. Bramble, T. V. Kolev, J. E. Pasciak, The approximation of the Maxwell eigenvalue problem using a least-squares method, Math. Comp. 74 (2005) 1575-1598. 


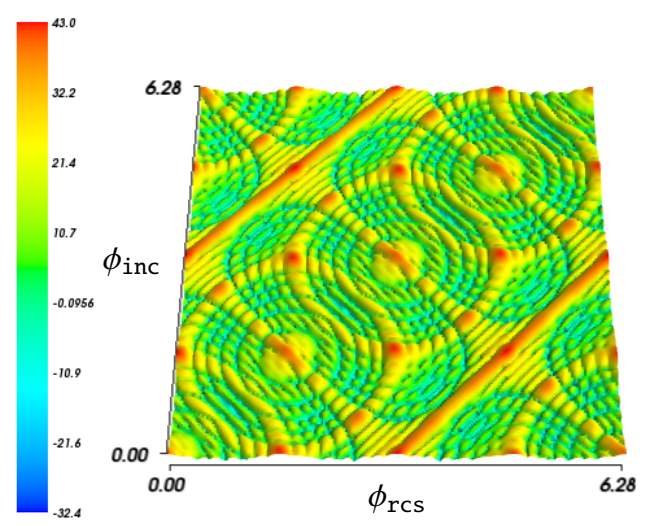

(a) $k=1$

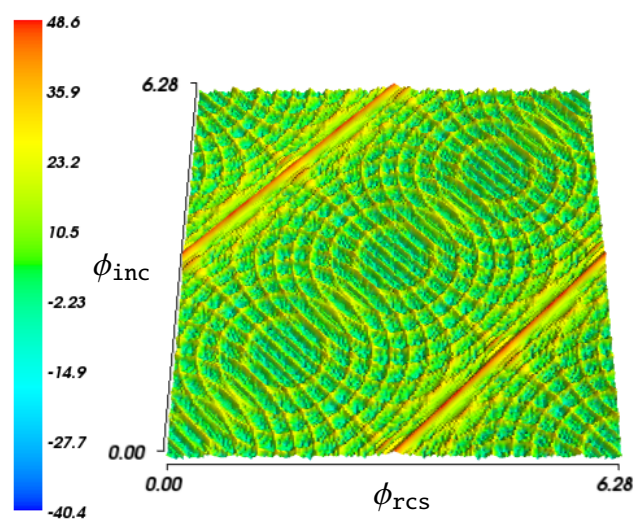

(c) $k=4$

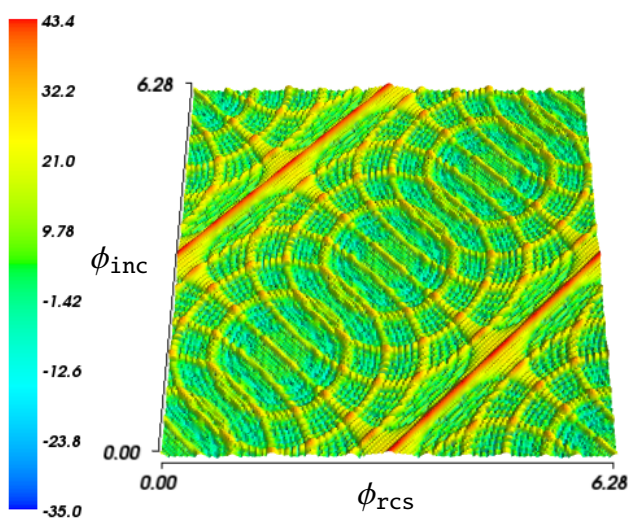

(b) $k=2$

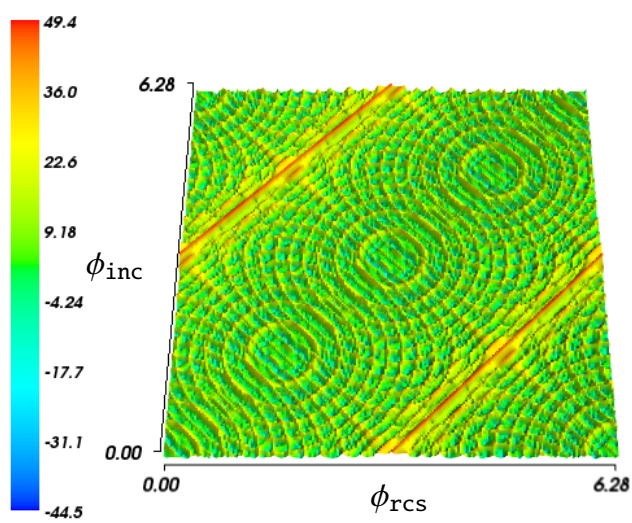

(d) $k=5$

Figure 7: (Example 4.) The RCS, measured in the directions $\theta_{\mathrm{rcs}}=\frac{\pi}{2}, \phi_{\mathrm{rcs}} \in[0,2 \pi]$, for a $6 \times 6$-lattice of metallic unit spheres for an incident plane wave of with angles $\theta_{\text {inc }}=\frac{\pi}{2}, \phi_{\text {inc }} \in[0,2 \pi]$ and different wavenumbers $k=1,2,4,5$.

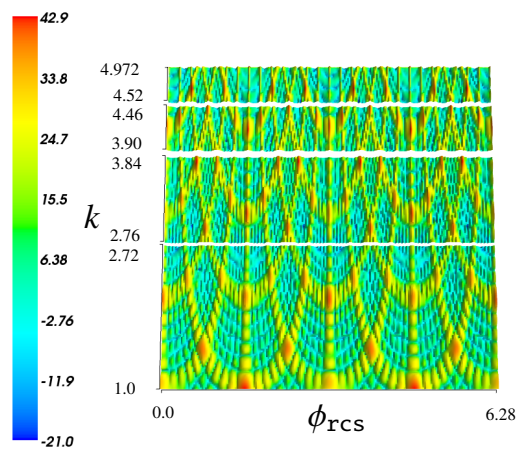

(a) $\theta=0$

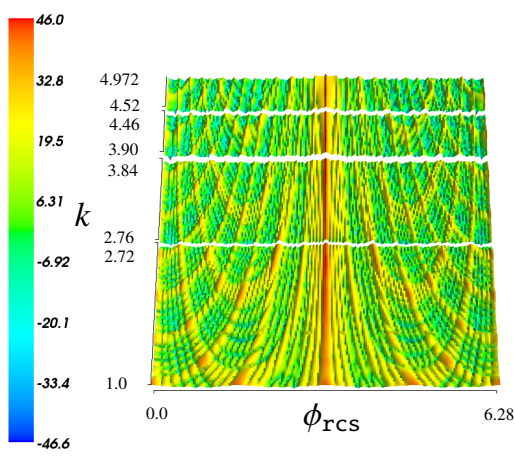

(b) $\theta=\frac{\pi}{2}$

Figure 8: (Example 4.) The RCS, measured in the directions $\theta_{\mathrm{rcs}}=\frac{\pi}{2}, \phi_{\mathrm{rcs}} \in[0,2 \pi]$, for a $6 \times 6$-lattice of metallic unit spheres for an incident plane wave of angles $\theta=0$ (a) and $\theta=\frac{\pi}{2}$ (b), $\phi=0$ and varying wavenumwbers $k \in[1,4.972]$. 


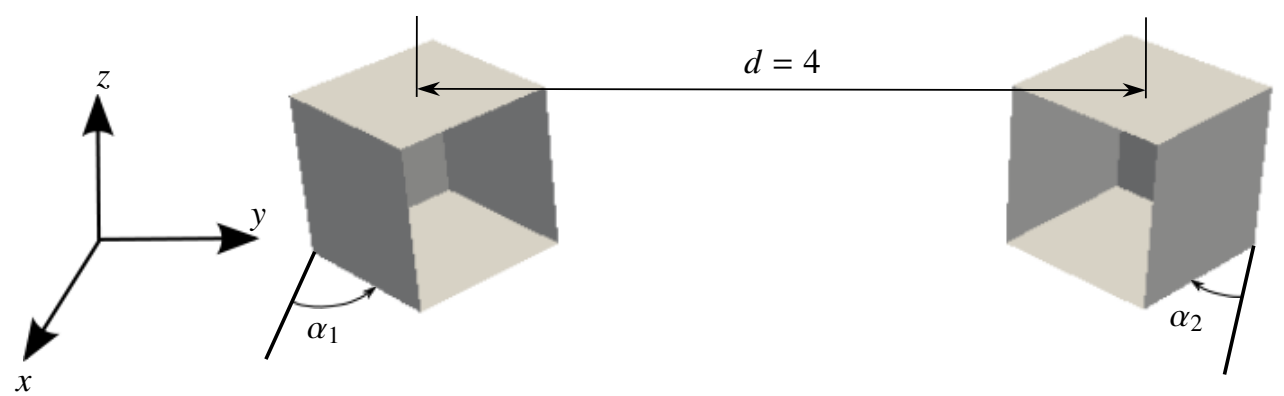

Figure 9: (Example 5.) Setup for scattering by two open metallic cavities.

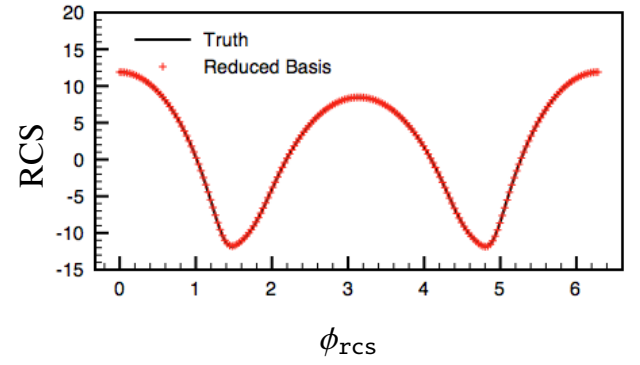

(a)

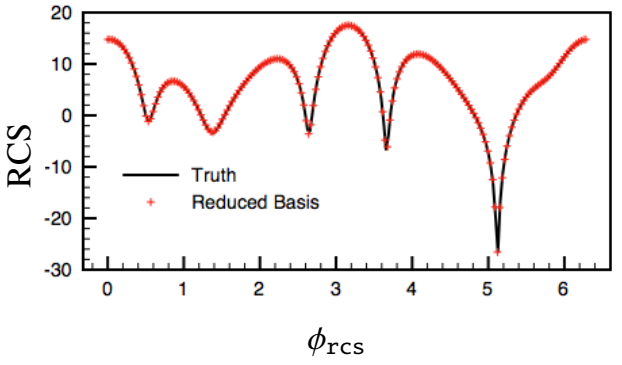

(b)

Figure 10: (Example 5.) Accuracy of the reduced basis RCS for randomly chosen parameters $k=1.395, \alpha_{1}=0.7552$ with a fixed $\alpha_{2}=\alpha_{1}$ (left) and for individually rotated random parameters $\alpha_{1}=1.5396, \alpha_{2}=1.1299$, for a fixed $k=2.491$ (right).
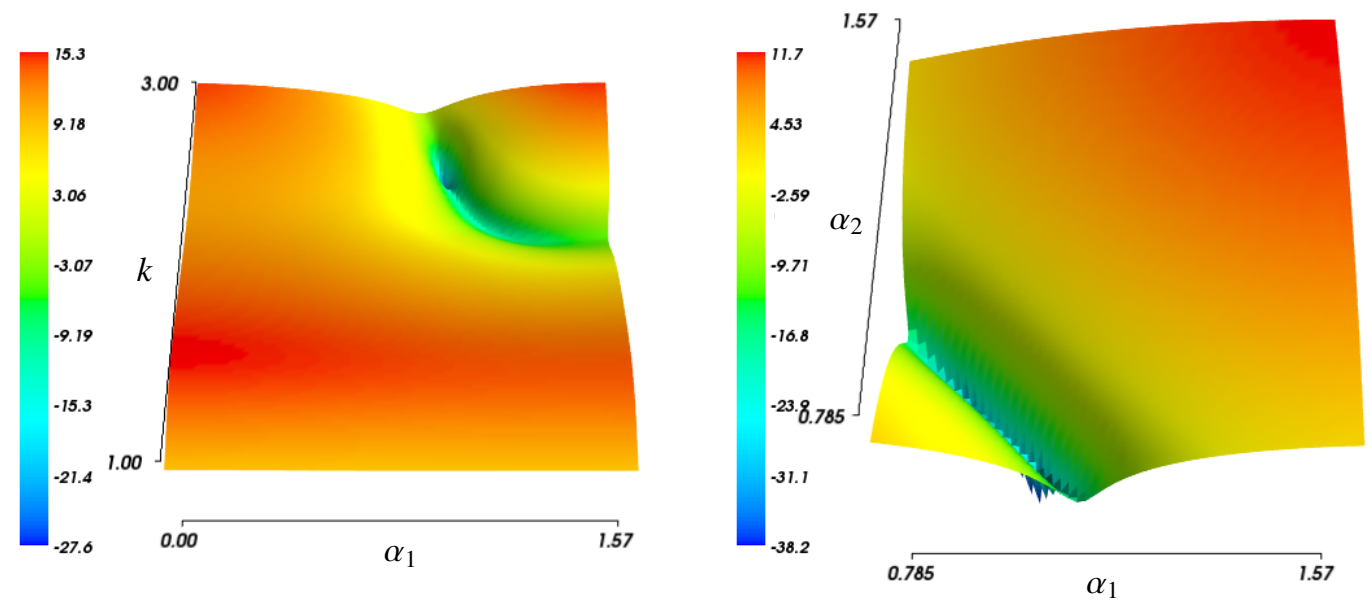

(a) The case of two metallic open cavities turned by an equal an- (b) The case of two metallic open cavities turned by an angle gle $\alpha \in\left[0, \frac{\pi}{2}\right]$ resp. $-\alpha$ around the $z$-axis for varying wavenum- $\alpha_{1} \in\left[\frac{\pi}{4}, \frac{\pi}{2}\right]$ resp. $-\alpha_{2} \in\left[\frac{\pi}{4}, \frac{\pi}{2}\right]$ around the $z$-axis for a fixed bers $k \in[1,3]$. wavenumber $k=2.78$.

Figure 11: (Example 5.) Monostatic RCS (with $\theta_{\text {inc }}=\theta_{\mathrm{rcs}}=\pi / 2, \phi_{\text {inc }}=\phi_{\mathrm{rcs}}=0$ ) for a dynamic configuration with two open metallic cavities. 


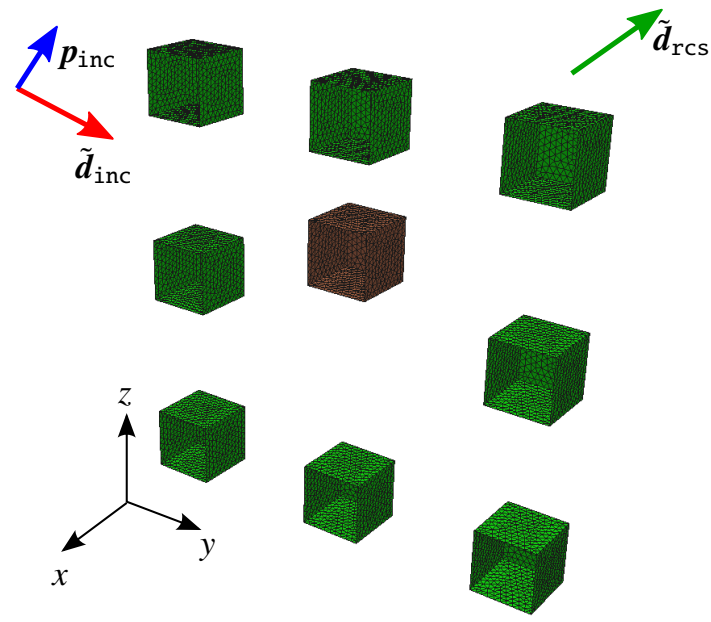

Figure 12: (Example 6.) A stochastic scattering configuration with in which the vertical location of the center cavity is assumed to be specified by a continuous uniformly distributed distribution in $[-1,1]$. 


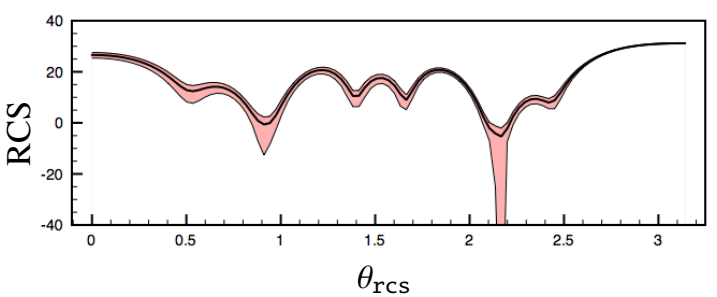

(a)

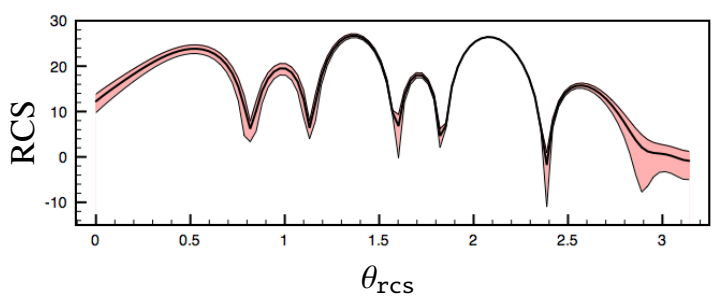

(c)

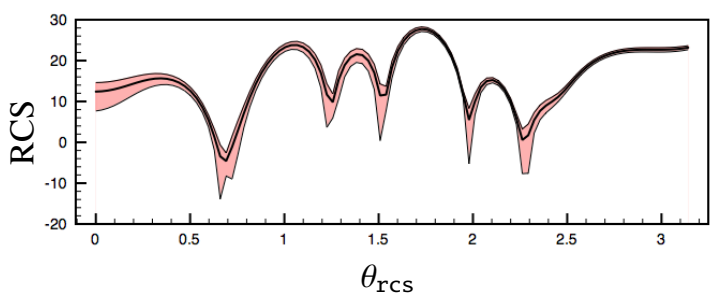

(b)

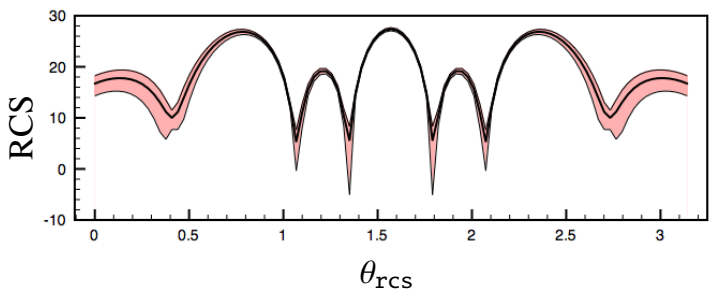

(d)

Figure 13: (Example 6.) Expected value of monostatic RCS and the interval $[E-\sqrt{V}, E+\sqrt{V}]$ for a stochastic 9 metallic box configuration for four different angles of incidence $\theta_{\text {inc }}=0, \frac{\pi}{6}, \frac{\pi}{3}, \frac{\pi}{2}$ (left to right), with $\phi_{\text {inc }}=0$.
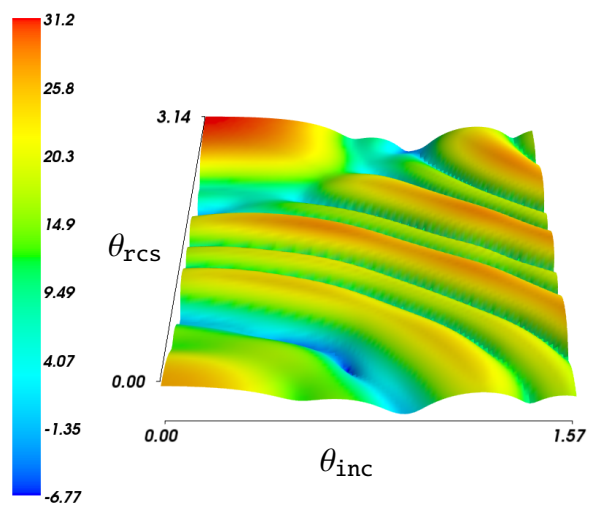

(a) Expected value of RCS.

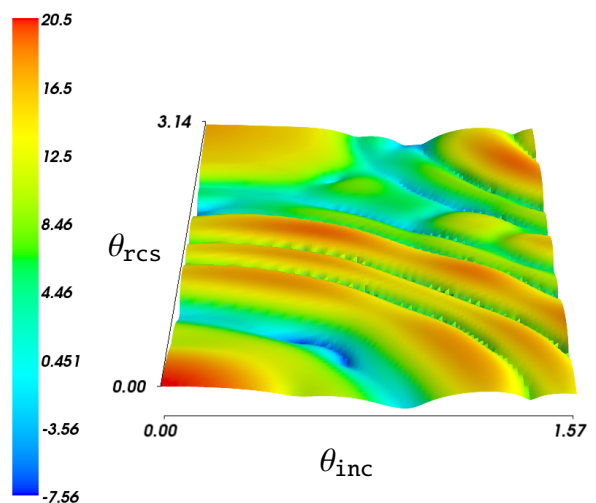

(b) Standard deviation of RCS

Figure 14: (Example 6.) Expected value and standard deviation of the RCS with respect to the incident and observed angles, for an uncertain 9 metallic box configuration. 


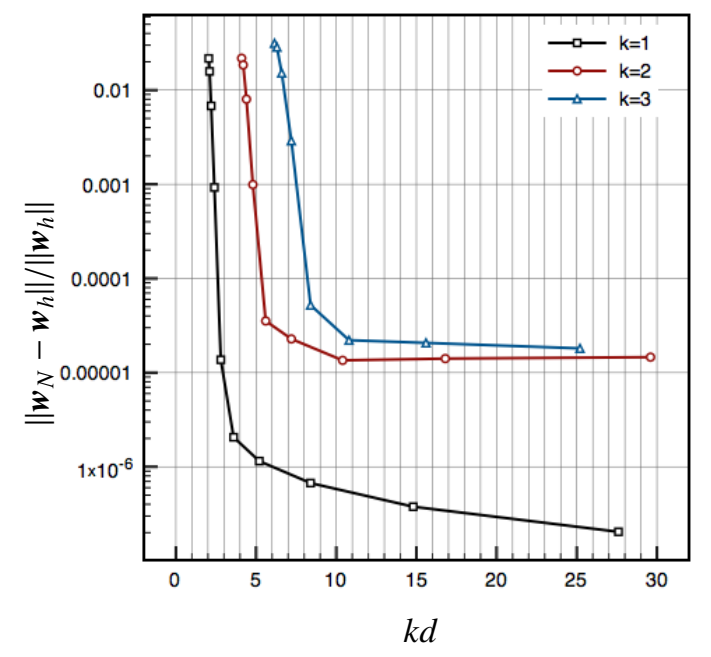

(a) Relative error

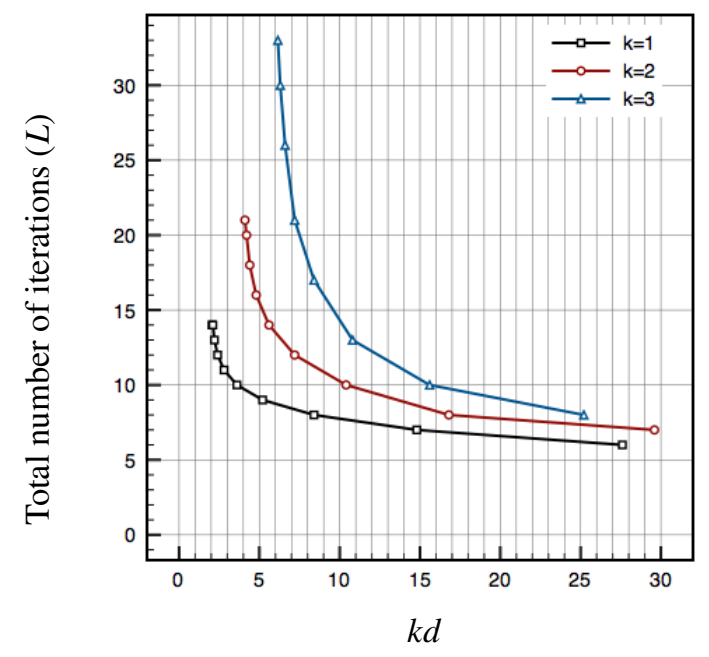

(b) Nubmer of iterations

Figure 15: (Example 7 .) Relative error and number of iterations for the two obstacle case with one sphere and one open cavity.

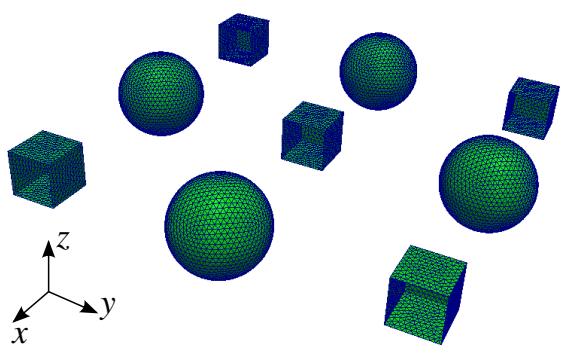

(a) Unperturbed geometry

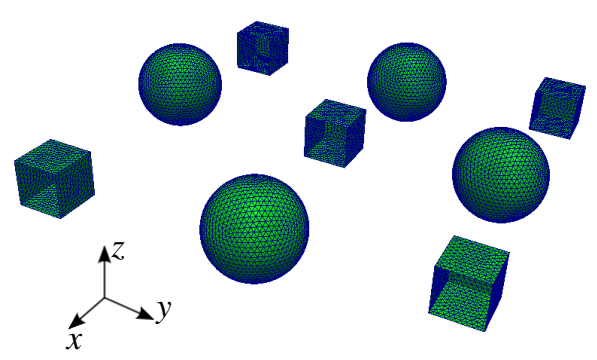

(b) Perturbed geometry

Figure 16: (Example 7 .) Geometrical setting used to simulate the case of a configuration using two different reference shapes. 


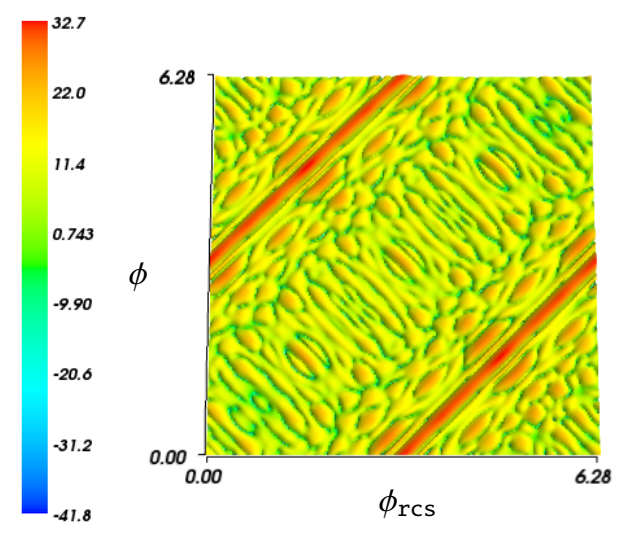

(a) RCS without perturbed geometry.

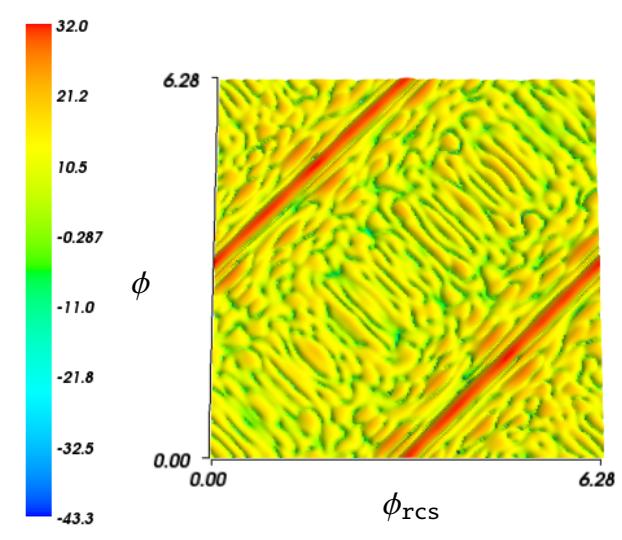

(b) RCS with perturbed geometry.

Figure 17: (Example 7 .) The RCS, measured in the directions $\theta_{\mathrm{rcs}}=\frac{\pi}{2}, \phi_{\mathrm{rcs}} \in[0,2 \pi]$, for a $3 \times 3$-lattice of metallic unit spheres and open cavities for an incident plane wave with angles $\theta_{\mathrm{inc}}=\frac{\pi}{2}, \phi_{\mathrm{inc}} \in[0,2 \pi]$ and fixed wavenumber $k=3$, for the unperturbed lattice (a) and for a perturbed lattice (b) 